\title{
AGOPUNTURA NELLA PREVENZIONE, TRATTAMENTO DELLE LESIONI E MIGLIORAMENTO DELLE PRESTAZIONI NEGLI ATLETI: REVISIONE DELLA LETTERATURA
}

\section{RECENSIONE ARTICOLO}

JUNIOR, Julio Cesar Dias ${ }^{1}$

Junior, Julio Cesar Dias. Agopuntura nella prevenzione, trattamento delle lesioni e miglioramento delle prestazioni negli atleti: Revisione della letteratura. Revista Científica Multidisciplinar Núcleo do Conhecimento. anno 04, Ed. 10, Vol. 10, pp. 5998. nell'ottobre 2019. ISSN: 2448-0959, Collegamento di accesso: https://www.nucleodoconhecimento.com.br/salute/agopuntura-inprevenzione

\section{RIEPILOGO}

Gli studi hanno dimostrato che l'agopuntura migliora le prestazioni fisiche negli atleti professionisti, ma il grande problema sono gli infortuni sportivi, che possono lasciarli Iontano dalle attività per lunghi periodi. Lo studio ha condotto una revisione della

${ }^{1}$ Laurea in Fisioterapia presso il Centro Universitario di Araraquara - UNIARA - 2005 (Araraquara-SP); Lato sensu studi post-laurea in Fisioterapia Ortopedica e Traumatologica dell'Istituto Cohen - Ortopedia, Riabilitazione e Medicina dello Sport 2006 (San Paolo-SP)Formazione in Terapia Manuale Osteopatica da parte dell'Istituto Cefisa - 2008 (Aquarraraa-SP); Miglioramento Professionale nella Rieducazione Posturale Globale e Riprogrammazione Motoria Sensoriale - RPG/RSM dell'Istituto Paulista di Studi Sistemici - IPES - 2010 (Ribeiro Preto-SP); Studi di laurea di Lato sensu in agopuntura sistemica presso I'Istituto Paulista di Studi Sistemici - IPES - 2013 (Ribeir preto-SP); Laurea magistrale post-laurea Stricto sensu in sviluppo territoriale e ambiente presso il Centro Universitario di Araraquara - UNIARA - 2016 (Araraquara SP); Miglioramento Professionale in Pilates Clinici e Funzionali dell'Istituto Paulista di Studi Sistemici - IPES - 2016 (Ribeir preto-SP). 
letteratura, alla ricerca dell'efficacia della tecnica nella prevenzione, nel miglioramento delle prestazioni e nel trattamento delle lesioni. Alcuni autori esemplificano e chiariscono il processo di neurofisiologia del dolore e il modo in cui l'agopuntura può intervenire in questo problema, altri, sviluppano e applicano protocolli per ottenere un miglioramento delle prestazioni, del trattamento delle malattie e della prevenzione delle malattie Lesione. Tra i risultati sono stati trovati ottantanove articoli sull'argomento, dove: ventidue hanno riferito il trattamento delle lesioni e trentasette la prevenzione e il miglioramento delle prestazioni atletiche, dirigendo l'applicazione una volta nella settimana, di 20 - 30 minuti, presentando risultati soddisfacenti per i dati analizzati. Sulla base di questa letteratura e pratica basata su prove si può concludere che l'agopuntura è un grande intervento per raggiungere questi obiettivi all'interno del settore dello sport.

Parole chiave: agopuntura, lesioni, dolore, prestazioni.

\section{INTRODUZIONE}

Per diversi millenni l'agopuntura è stata utilizzata come forma di prevenzione e trattamento di varie malattie del corpo. Questa tecnica della medicina cinese manipolò aghi di pietre e brufoli di pesce per tutta l'età della pietra (3000 anni a.C) ${ }^{1,2} 2||$ classico imperatore giallo: Huang Di Nei Jing, costituiva uno dei più antichi documenti della medicina tradizionale cinese (MTC), integrando ai giorni nostri le basi filosofiche, che concettualizzano l'equilibrio dell'universo e della natura, che nella sua visione (cinese), l'energia si chiama Chi o Qi. Questo fattore ha due principi antagonisti che vengono completati e tendono ad essere in equilibrio, anche se è sotto l'influenza dell'universo, chiamato Yin e Yang. ${ }^{3}$

L'agopuntura è definita come l'armonizzazione dell'individuo con l'ambiente in cui egli è, non solo, di inserire aghi e porre rimedio al problema della malattia, ma di raggiungere l'ideale di prevenzione delle varie patologie. ${ }^{4,5} \mathrm{E}$ una procedura eseguita con obiettivo terapeutico, non è tossico, senza l'uso di elementi chimici, con effetti collaterali minimi, anche se è in grado di rilasciare sostanze endogene come i neurotrasmettitori. $4,6,7,8,9$ 
L'intervento è quello di stimolare gli acupoint per ottenere l'effetto terapeutico desiderato e raggiungere l'omeostasi dell'organismo. Molti studi esemplificano gli effetti della teoria neurale non oppioide (inibisce gli impulsi nervosi che agiscono sul sistema nervoso periferico e centrale); teoria emorale (stimola il rilascio di oppioidi endogeni, altri ormoni e neurotrasmettitori). ${ }^{10}{ }^{11}$ Scopo nel controllo del dolore, l'associazione di aghi con corrente elettrica, chiamato elettroagocupuntura, potenziati e presenta benefici nel pratica clinica, oltre ad essere utilizzato anche come: agopuntura auricolare; teschio puntura; moxabustào; ventose di aspirazione; agopuntura laser. 3,10

Nello sport, l'uso della tecnologia alla ricerca di risultati migliori continua a crescere, superando i limiti del corpo umano. Diverse terapie naturali o integrative sono studiate come fonte di terapia complementare per migliorare le condizioni atletiche. Anche se il numero di studi è scarso, l'accettazione dell'agopuntura è ancora molto curiosa, dal momento che molti individui dubitano ancora delle prove scientifiche. ${ }^{9}$ Tuttavia, il suo uso nell'ambiente sportivo è aumentato, agendo nel trattamento del dolore e delle lesioni atletiche, accelerando il processo di recupero dell'individuo. Un altro problema presentato è l'intervento efficace nel migliorare le prestazioni fisiche e prevenire gli infortuni da parte di atleti dilettanti e professionisti. 12, 13, 14

\section{GOL}

L'obiettivo di questo lavoro era quello di condurre un'indagine bibliografica sull'uso dell'agopuntura nella prevenzione, nel miglioramento delle prestazioni atletiche e nel trattamento delle lesioni in varie modalità sportive.

\section{METODOLOGIA}

Un'indagine bibliografica è stata condotta nei database Scielo, Google Acad-mico e Scoopus, per ottenere informazioni su: agopuntura nella prevenzione, miglioramento delle prestazioni e nel trattamento delle lesioni sportive. Sono state utilizzate le seguenti parole chiave: agopuntura; agopuntura e dolore; agopuntura nella 
prevenzione; lesioni sportive e agopuntura; agopuntura e miglioramento delle prestazioni atletiche; trattamento delle lesioni e dell'agopuntura.

Per delimitare il ritaglio di questo studio, è stata eseguita una prima proiezione tra $\mathrm{i}$ numerosi articoli trovati, per un totale di 89 manoscritti, selezionando 22 che hanno affrontato il soggetto di agopuntura nel trattamento delle lesioni e 37 affrontando la prevenzione delle lesioni sportive e migliorare le prestazioni atletiche. Sulla base di questa analisi, vale la pena ricordare che di tutti gli studi trovati principalmente, I'universo della ricerca comprendeva 59 articoli pubblicati su riviste scientifiche, tenute congressuali, libri, siti web, che nel titolo, astratti o parole chiave terminologie utilizzate durante la ricerca.

\section{RISULTATI E DISCUSSIONE}

In questa ricerca ha prodotto diversi articoli (grafico 1; tabella 1), essendo stato eseguito in isolamento attraverso le parole chiave.

Grafico 1: numero totale di articoli trovati e utilizzati nella ricerca.

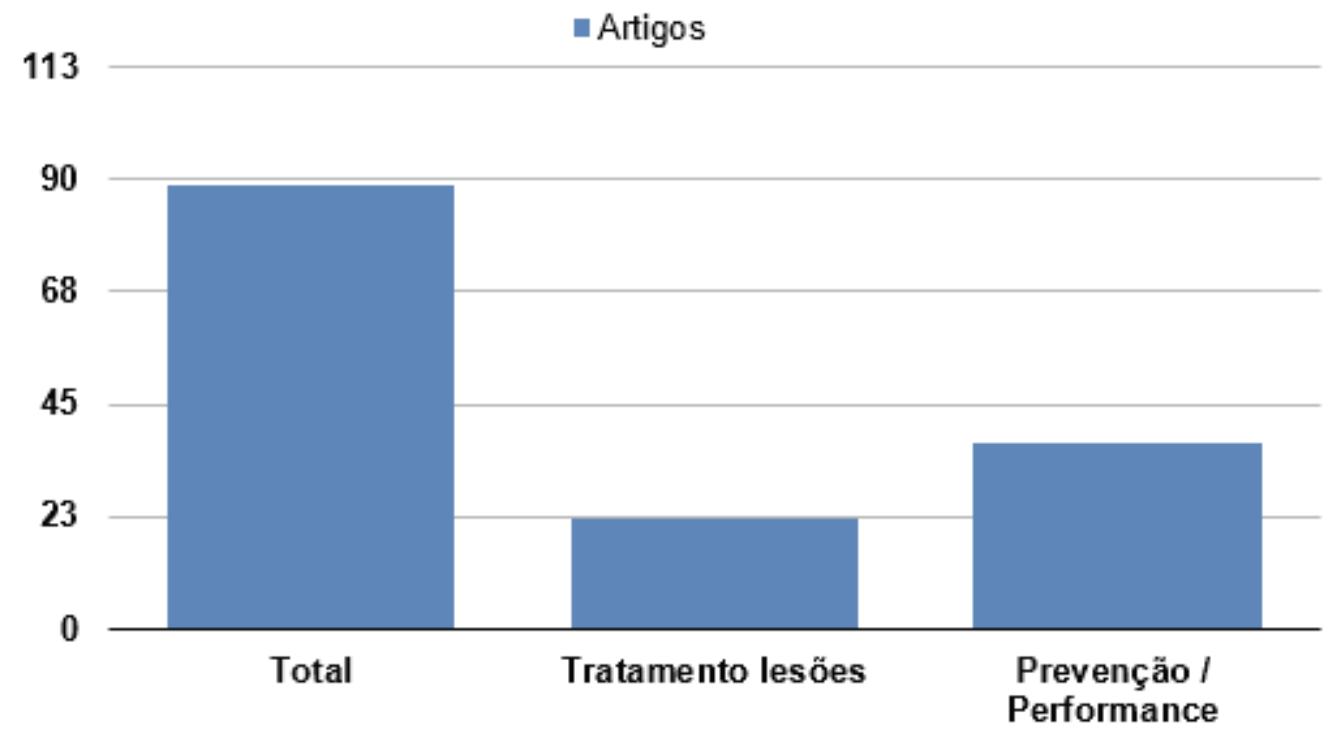


Tabella 1: Articoli relativi alla prevenzione, al miglioramento delle prestazioni atletiche e al trattamento delle lesioni sportive.

\begin{tabular}{|c|c|c|}
\hline Autori & Titolo articolo & Giornali/Anais/Congressi/Siti \\
\hline $\begin{array}{l}\text { Ehrlich D, Haber } \\
\text { P, } 1992\end{array}$ & $\begin{array}{l}\text { Influence of } \\
\text { acupuncture on pysical } \\
\text { performance capacity } \\
\text { and Haemodynamic } \\
\text { Parameters }\end{array}$ & J. Sports Med \\
\hline $\begin{array}{lr}\text { Santos } & \text { VC, } \\
\text { Kawano } & \text { MM, } \\
\text { Banja RA, 2008 }\end{array}$ & $\begin{array}{l}\text { Acupuntura na melhora } \\
\text { da performance em } \\
\text { atletas juvenis de } \\
\text { handebol }\end{array}$ & Rev Saúde e Pesq \\
\hline $\begin{array}{l}\text { Rubio K, Godoy } \\
\text { Moreira F, } 2008\end{array}$ & $\begin{array}{l}\text { A dor em corredores } \\
\text { com fascite plantar: o } \\
\text { uso da acupuntura }\end{array}$ & Rev Dor \\
\hline $\begin{array}{ll}\text { Pires } & \text { TF, } \\
\text { Pellegrinotti } & \text { IL, } \\
2010 & \end{array}$ & $\begin{array}{l}\text { Acupuntura na } \\
\text { Performance Atlética: } \\
\text { Estudo Exploratório }\end{array}$ & 8ª Mostra Acadêmica da UNIMEP \\
\hline $\begin{array}{l}\text { Yang HY, Liu TY, } \\
\text { Gao M, } 2006\end{array}$ & $\begin{array}{l}\text { Electrical acupoint } \\
\text { stimulation increases } \\
\text { athletes rapid strength }\end{array}$ & Zhongguo Zhen Jiu \\
\hline $\begin{array}{ll}\text { Luna } & \text { MP, } \\
\text { Fernandes } & \text { Filho } \\
\text { J, } 2005 & \end{array}$ & $\begin{array}{l}\text { Efeitos da Acupuntura } \\
\text { na performance de } \\
\text { Atletas velocista de alto } \\
\text { rendimento do Rio de } \\
\text { Janeiro }\end{array}$ & Fit e Perform J \\
\hline $\begin{array}{l}\text { Dias Junior JC, } \\
\text { Marino DM, } 2019\end{array}$ & $\begin{array}{l}\text { Acupuntura na } \\
\text { prevenção de lesões } \\
\text { musculares em atletas } \\
\text { de futebol profissional }\end{array}$ & Rev Fisiot S Fun \\
\hline
\end{tabular}




\begin{tabular}{|c|c|c|}
\hline Maciocia G, 2007 & $\begin{array}{l}\text { Os fundamentos da } \\
\text { medicina chinesa: um } \\
\text { texto abrangente para } \\
\text { acupunturistas } \\
\text { fitoterapeutas }\end{array}$ & Libro \\
\hline $\begin{array}{ll}\text { Akimoto } & \text { T, } \\
\text { Nakahori } & \text { C, } \\
\text { Aizawa } & \text { K, } \\
\text { Kimura } & \text { F, } \\
\text { Fukubayashi } & \text { T, } \\
\text { Kono I, 2003 } & \end{array}$ & $\begin{array}{l}\text { Acupuncture and } \\
\text { responses of imunoligic } \\
\text { and endocrine markers } \\
\text { during competition }\end{array}$ & Med Sci in Sports Exerc \\
\hline $\begin{array}{lr}\text { Karvelas } & \text { BR, } \\
\text { Hoffman } & \text { MD, } \\
\text { Zeni Al, } 1996\end{array}$ & $\begin{array}{l}\text { Acute Effects of } \\
\text { Acupuncture on } \\
\text { Physiological and } \\
\text { Psychological } \\
\text { Responses to Cycle } \\
\text { Ergometry }\end{array}$ & Arch Phys Med Rehabil \\
\hline $\begin{array}{l}\text { Fry AC, Kraemer } \\
\text { KJ, } 1997\end{array}$ & $\begin{array}{l}\text { Resistance exercise ov } \\
\text { ertraing } \\
\text { and overreaching: neur } \\
\text { oendocrine responses }\end{array}$ & Sports Med \\
\hline KnardahI S, 1998 & $\begin{array}{l}\text { Sympathetic } \\
\text { nerve activity after } \\
\text { acupuncture in humans }\end{array}$ & $\begin{array}{l}\text { Department } \quad \text { of } \\
\text { Neurophysiology }\end{array}$ \\
\hline $\begin{array}{l}\text { Barlas PJ, } \\
\text { Robinson JA, Ba } \\
\text { xter GD, } 2000\end{array}$ & $\begin{array}{l}\text { Lack ofeffect of acupun } \\
\text { cture upon signs } \\
\text { and symptoms of delay } \\
\text { ed } \\
\text { onset muscle soreness }\end{array}$ & Clinical Physio \\
\hline $\begin{array}{l}\text { Rossetto SC, } \\
2009\end{array}$ & $\begin{array}{l}\text { Acupuntura nos } \\
\text { Esportes }\end{array}$ & Libro \\
\hline
\end{tabular}




\begin{tabular}{|c|c|c|}
\hline $\begin{array}{l}\text { França D, } \\
\text { Fernandes- } \\
\text { Senna V, Cortez } \\
\text { CM, } 2004\end{array}$ & 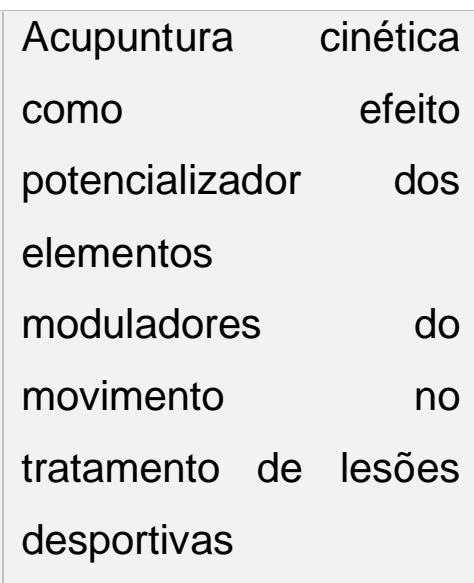 & Fisioter Bras \\
\hline $\begin{array}{l}\text { Wadsworth L T, } \\
2006\end{array}$ & $\begin{array}{l}\text { Acupuncture in sports } \\
\text { medicine }\end{array}$ & Curr Sports Med Rep \\
\hline Barela J A, 2000 & $\begin{array}{l}\text { Estratégias de Controle } \\
\text { em Movimentos } \\
\text { Complexos: Ciclo } \\
\text { Percepção - Ação no } \\
\text { Controle Postural }\end{array}$ & Rev Paul de Educ Fís \\
\hline $\begin{array}{l}\text { Papler PG et al., } \\
1999\end{array}$ & $\begin{array}{l}\text { Reabilitação do joelho. } \\
\text { In: Greve J.M. A. e } \\
\text { Amattuzzi, } \\
\text { Medicina M.M. } \\
\text { reabilitação aplicada à } \\
\text { ortopedia } \\
\text { traumatologia }\end{array}$ & Libro \\
\hline $\begin{array}{l}\text { Gemeo LH. } \\
\text { Ignatti C, } 2004\end{array}$ & $\begin{array}{l}\text { Acupuntura como } \\
\text { Ferramenta Auxiliar do } \\
\text { Aumento da } \\
\text { Performance } \\
\text { Desportiva. In: Anais do } \\
\text { Simpósio Internacional } \\
\text { de Ciências Integradas } \\
\text { da UNAERP }\end{array}$ & Anais Simpósio \\
\hline
\end{tabular}




\begin{tabular}{|c|c|c|}
\hline Frasca L, 2011 & $\begin{array}{l}\text { Desempenho na Ponta } \\
\text { da Agulha }\end{array}$ & Rev Farmac \\
\hline Costa V, 2013 & $\begin{array}{lr}\text { Acupuntura } & \text { Previne } \\
\text { Lesões e ajuda a } & \\
\text { melhorar } & \text { o } \\
\text { Desempenho } & \text { nas } \\
\text { Pistas [periódico na } \\
\text { internet] }\end{array}$ & $\begin{array}{l}\text { http://blogs.oglobo.globo.com/pulso/ } \\
\text { post/acupuntura-previne-lesoes- } \\
\text { ajuda-melhorar-desempenho-nas- } \\
\text { pistas-500798.html. }\end{array}$ \\
\hline Luna M, 2016 & $\begin{array}{l}\text { Os Benefícios da } \\
\text { Acupuntura no Esporte }\end{array}$ & $\begin{array}{l}\text { http://www.ibramrp.com.br/noticia/49 } \\
\text { /os-beneficios-da-acupuntura-no- } \\
\text { esporte }\end{array}$ \\
\hline $\begin{array}{l}\text { Bopp-Limoge C, } \\
1998\end{array}$ & $\begin{array}{l}\text { L’acupuncture Permet- } \\
\text { elle D`ameliorer les } \\
\text { Performances } \\
\text { Sportives Stude } \\
\text { Personnelle a Propos } \\
\text { de } 35 \text { Sportifs de haut } \\
\text { niveau. }\end{array}$ & These Medicine \\
\hline Lin ZP et al., 2009 & $\begin{array}{l}\text { Effects of acupuncture } \\
\text { stimulation on recovery } \\
\text { ability for male elite } \\
\text { basketball athletes }\end{array}$ & Amer Jour of Chin Med \\
\hline $\begin{array}{l}\text { Lin, ZP et al., } \\
2009\end{array}$ & $\begin{array}{l}\text { Effect of auricular } \\
\text { acupuncture on oxygen } \\
\text { consumption of boxing } \\
\text { athletes }\end{array}$ & Chin Med Jour \\
\hline $\begin{array}{l}\text { Dhillon S et al., } \\
2008\end{array}$ & $\begin{array}{l}\text { The acute effect of } \\
\text { acupuncture on } 20-\mathrm{km} \\
\text { cycling performance. }\end{array}$ & Clin Jour of Spo Med \\
\hline $\begin{array}{l}\text { Hubscher M et } \\
\text { al., } 2010\end{array}$ & $\begin{array}{l}\text { Immediate effects of } \\
\text { acupuncture }\end{array}$ & Euro Jour of Appli Physi \\
\hline
\end{tabular}




\begin{tabular}{|c|c|c|}
\hline & $\begin{array}{l}\text { strength performance: a } \\
\text { ramdomized, controlled } \\
\text { crossover trial }\end{array}$ & \\
\hline $\begin{array}{l}\text { Geng } L J \text { et al., } \\
1995\end{array}$ & $\begin{array}{l}\text { Investigation on the } \\
\text { effects of ear } \\
\text { acupressure on } \\
\text { exercise-induced lactic } \\
\text { acid levels and the } \\
\text { implications for athletic } \\
\text { training }\end{array}$ & Amer Jour of Acupu \\
\hline Franco RS, 2012 & $\begin{array}{l}\text { Avaliação do Efeito da } \\
\text { Acupuntura sobre o } \\
\text { Desempenho Físico } \\
\text { pelo Teste do Banco de } \\
\text { Harvard }\end{array}$ & Dissertação \\
\hline $\begin{array}{l}\text { Fonseca LP, } \\
\text { Lessa JFM, } 2011\end{array}$ & $\begin{array}{l}\text { Efeito da Aplicação da } \\
\text { Acupuntura na } \\
\text { Resistência Muscular } \\
\text { Localizada de Membros } \\
\text { Superiores de } \\
\text { Praticantes } \\
\text { Exercício Resistido. }\end{array}$ & Mobografia \\
\hline $\begin{array}{lr}\text { Belmiro } & \text { H, } \\
\text { Vicentini } & \text { D, } \\
\text { Camilotti } & \text { CM, } \\
2013 & \end{array}$ & $\begin{array}{l}\text { Efeitos da Acupuntura } \\
\text { no Desempenho Motor } \\
\text { de Atletas. }\end{array}$ & Rev Fac Educ Fis \\
\hline $\begin{array}{l}\text { Rubio K; Godoy } \\
\text { Moreira F, } 2007\end{array}$ & $\begin{array}{l}\text { A representação da dor } \\
\text { em atletas olímpicos } \\
\text { brasileiros }\end{array}$ & Rev Dor \\
\hline Parisotto D, 2014 & $\begin{array}{ll}\text { Efeito Imediato } & \mathrm{da} \\
\text { Aplicação } & \mathrm{da}\end{array}$ & Dissertação \\
\hline
\end{tabular}




\begin{tabular}{|c|c|c|}
\hline & $\begin{array}{l}\text { Acupuntura na Dor } \\
\text { Muscular Tardia e na } \\
\text { Capacidade de } \\
\text { Contração Muscular }\end{array}$ & \\
\hline Staud R, 2007 & $\begin{array}{l}\text { Mechanisms of } \\
\text { acupunture analgesia: } \\
\text { effective therapy for } \\
\text { musculoskeletal pain? }\end{array}$ & Curr rheumatol Rep \\
\hline $\begin{array}{lr}\text { Bucinskaite } & V \text {, } \\
\text { Lundeberg } & T, \\
\text { Stenfors } \quad C, \\
\text { Ekblom A, Dahlin } \\
\text { L, Theodorsson } \\
\text { E, } 1994\end{array}$ & $\begin{array}{l}\text { Effects of electro- } \\
\text { acupuncture and } \\
\text { pysical exercice on } \\
\text { regional concentrations } \\
\text { of neuropeptides in rat } \\
\text { brain }\end{array}$ & Brain research \\
\hline Shang C, 2009 & $\begin{array}{l}\text { Prospective tests on } \\
\text { biological models of } \\
\text { acupuncture }\end{array}$ & $\begin{array}{l}\text { Evid Based Complement Alternat } \\
\text { Med. }\end{array}$ \\
\hline Bowsher D,1988 & $\begin{array}{l}\text { Mechanisms of } \\
\text { acupuncture. In: Filshie } \\
\mathrm{J} \text {, White A. editors. } \\
\text { Medial Acupuncture: a } \\
\text { western scientific } \\
\text { approach. }\end{array}$ & Libro \\
\hline Ma SX, 2004 & $\begin{array}{l}\text { Neurobiology } \\
\text { acupuncture: } \\
\text { CAM }\end{array}$ & $\begin{array}{l}\text { Evid Based Complement Alternat } \\
\text { Med }\end{array}$ \\
\hline $\begin{array}{l}\text { Li J, Wang Q, } \\
\text { Liang H, Dong H, } \\
\text { Li Y, Ng EH, et al., } \\
2012\end{array}$ & $\begin{array}{l}\text { Biophysical } \\
\text { characteristics of } \\
\text { meridians and } \\
\text { acupoints: a systematic } \\
\text { review. }\end{array}$ & $\begin{array}{l}\text { Evid Based Complement Alternat } \\
\text { Med. }\end{array}$ \\
\hline
\end{tabular}


Lee, SH, Chung, Effects Acupunturaq on Neurocienses Letters

$\mathrm{SH}$, Lee, JS, Kim, the 5-

SS, Shin, HD, hydroxytryptamine

Lim, BV, et al., synthesis and

2002 tryptophan hydroxylase

expression in dorsal in

the dorsal raphe of

exercice rats.

Zyloney CE, Imaging the functional Mol pain

Jensen K, Polich connectivity of the

G, Loiotile RE, Periaqueductal Gray

Cheetham A, during genuine and

LaViolette PS, et sham

al., 2010 electroacupuncture treatment.

Minori AET, Mejia Atuação da Acupuntura Monografia

DPM, 2007 para o Tratamento de LER/DORT no Ombro.

$\begin{array}{lrlr}\text { Brum KN, } & \text { Tratamento } & \text { de } \\ \text { Alonso, AC, } & \text { massagem } & \text { e } \\ \text { Brech GC, 2009 } & \text { acupuntura } & \text { em } \\ & \text { corredoresrecreacionai } \\ & \text { s com síndrome do } \\ & \text { piriforme }\end{array}$

Hongwen S, 2003 Clinical Observation on J Tradit Chin Med.

Acupuncture Treatment of Piriformis Syndrome.

Rocha TBX, Análise Comparativa Rev Bras Cien e Mov

Vilela Junior GB, Eletromiográfica do

Martins GC, Reto Femoral em

Isometria na Posição 


\begin{tabular}{|c|c|c|}
\hline $\begin{array}{l}\text { Manzatto L, } \\
\text { Grande AJ, } 2012\end{array}$ & $\begin{array}{lr}\text { Inferior } & \text { do } \\
\text { Agachamento } & \text { Wall } \\
\text { Slide, antes e } & \text { após a } \\
\text { Aplicação } & \text { da } \\
\text { Acupuntura no } & \text { Ponto } \\
\text { ST45 } & \end{array}$ & \\
\hline $\begin{array}{l}\text { Pinheiro RG, } \\
\text { Mejia DPM, } 2012\end{array}$ & $\begin{array}{l}\text { Efeito da Acupuntura na } \\
\text { Melhora do Paciente } \\
\text { com Quadro Álgico de } \\
\text { Lesão de Menisco } \\
\text { Medial. }\end{array}$ & Monografia \\
\hline $\begin{array}{l}\text { Nunes EA, Mejia } \\
\text { DPM, } 2012\end{array}$ & $\begin{array}{lr}\text { Tratamento } & \text { de } \\
\text { Acupuntura para } \\
\text { Combater Dores nos } \\
\text { Ombros. }\end{array}$ & Monografia \\
\hline Tolentino F, 2016 & $\begin{array}{l}\text { Efeito de um } \\
\text { Tratamento com } \\
\text { Auriculoterapia na Dor, } \\
\text { Funcionalidade e } \\
\text { Mobilidade de Adultos } \\
\text { com Dor Lombar } \\
\text { Crônica. }\end{array}$ & Dissertação \\
\hline
\end{tabular}

\section{ACUPUNCTURE NELLA PREVENZIONE DI FERITE}

La prevenzione degli infortuni nello sport è una grande battaglia da vincere da professionisti che lavorano con gli atleti. Nel calcio, le lesioni muscolari corrispondono al $20-40 \%$ di tutte le lesioni sportive, prevalentemente l'80-90\% negli arti inferiori. ${ }^{15}$

L'infortunio muscolare è il grande cattivo di tutti gli atleti, da vari sport, e può lasciarli lontano dalle loro attività per un lungo periodo. Pertanto, è interessante presentare una proposta di intervento profilattico, portando l'idea di una tecnica per prevenire o ridurre 
i rischi di questo tipo di lesioni negli atleti. In questo caso l'agopuntura si dimostra una tecnica benefica.

Nel suo studio, Dias Junior ${ }^{16}$, ha visto la partecipazione di 54 atleti maschi, appartenenti a una squadra di calcio professionistica. Sono stati distribuiti in 6 gruppi: gruppo 1 - agopuntura per riequilibrare il sistema, trovato attraverso la valutazione; gruppo 2 - che ha ricevuto il protocollo con punti specifici: milza pancreas 3, milza pancreas 6 (Figura 1A) e stomaco 36 (Figura 1B); gruppo 3 - stomaco 36 (Figura 1B); gruppo 4 - pancreas milza 6 (Figura 1A); gruppo 5 - agopuntura dell'orecchio; gruppo di controllo - non ha ricevuto alcun intervento. Dopo otto sessioni, una a settimana, $\mathrm{i}$ risultati hanno mostrato che i gruppi 1 e 2 non presentavano alcun tipo di infortunio; gruppo 4: due lesioni; gruppi 3 e 5: presentato 1 lesione; controllo: presentato 6 casi di problemi muscolari.

Figura 1 A: pancreas della milza 3 - sul lato mediale del piede, posttero-inferiore dell'articolazione metatarsale-falina, nella linea della giunzione della pelle scura e chiara; pancreas milza 6: 3 tsun sopra il maleolo mediale, sul bordo posteromediale della tibia; B: stomaco 36 - 3 tsun sotto la rotula tra il muscolo della tibia anteriore e il muscolo estensore lungo delle dita; stomaco 37 - 3 tsun al di sotto di zusanli (E36), sul lato anteriore laterale del muscolo della tibia; stomaco 38 - 8 tsun sotto il ginocchio, 2 tsun sotto il Point Shangjuxu (E37), sul lato del muscolo tibia anteriore; stomaco 41 nel punto medio dorsale della caviglia sopra il legamento crociato, tra i tendini del muscolo estensore lungo e salato e il lungo estensore delle dita.

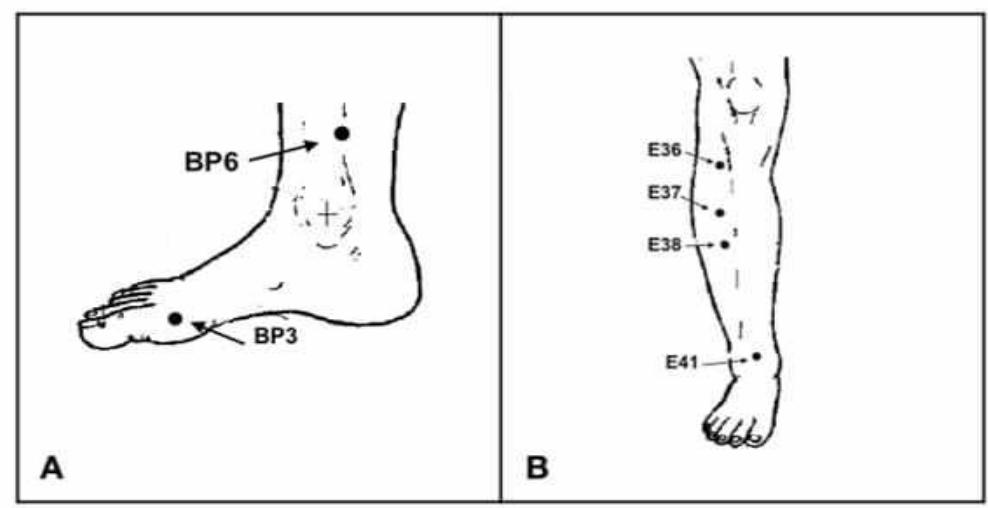

Fonte: Wen ${ }^{17}$ 
La milza del pancreas ha la funzione di estrarre energia dal cibo, distribuire al corpo e controllare il sangue all'interno dei vasi sanguigni. II forte Qi che presenta sarà trasportato a tutti i muscoli del corpo, in particolare agli arti, ma se il Qi è in deficit, l'energia non verrà trasmessa al tessuto muscolare e l'atleta presenterà affaticamento e muscoli indeboliti. ${ }^{18}$

Secondo Ross ${ }^{19}$, i punti di milza pancreas 3 e 6 sono associati con lo stomaco 36 da tonining sangue Qi. L'elemento terra è responsabile per l'equilibrio energetico e la disponibilità di Qi e sangue come fonti di energia per l'attività mentale, emotiva e fisica. THE MTC, riferisce che tonificando questo elemento, mantiene i muscoli nutriti da Qi e Xue (sangue in THE MTC), con l'obiettivo di migliorare le prestazioni atletiche. Questo mantiene l'obbligo di tonificare l'organo che produce Xue (Liver) e mantiene il fluido energetico (Spleen Pancreas e Rene), poiché la funzionalità del sangue è quella di nutrire il corpo, oltre a completare l'azione nutritiva di Qi.

L'allenamento muscolare, il rilassamento e la forza contrattile sono legati alla nutrizione del fegato xue. Se la nutrizione è carente, crampi e parestesia degli arti causeranno lesioni. D'altra parte la milza monitora lo xue all'interno dei vasi e rimuove qi dalla trasmissione di alimenti al corpo: Strong Qi, trasmetterà buona energia ai muscoli; un Qi indebolito si tradurrà in un muscolo stanco e debole. 19, 20

Sulla base di questa prova questa tecnica è praticata negli sport che presentano risultati rapidi, migliorando la qualità della forza muscolare, condizioni cardiorespiratorie, flessibilità e benessere mentale e fisico degli sportivi. 20, 21, 22 Yang e 22 dipendenti, ha raggiunto un significativo aumento della forza muscolare, fornendo un miglioramento della velocità degli atleti. A sua volta, Luna e collaboratori ${ }^{24}$, ha dichiarato che la stimolazione dei punti di agopuntura migliora le prestazioni sportive, plasticità muscolare prevenire lesioni.

Uno studio condotto in Giappone evidenzia l'effetto dell'agopuntura sul benessere fisico delle giocatrici di calcio durante la competizione. Sono stati divisi in due gruppi, dove uno è stato sottoposto al trattamento e l'altro controllo. I livelli di cortisolo (valutazione del sistema endocrino) e la valutazione del benessere fisico sono stati 
valutati utilizzando il questionario POMS (valuta lo stato fisico e mentale): è stato osservato un miglioramento del sistema immunitario ( ridotta secrezione $\mathrm{S} \lg \mathrm{A}$ ), miglioramento dello stress mentale (cortisolo in diminuzione) e migliora il benessere fisico, la flessibilità e la tensione muscolare. ${ }^{25}$

Tuttavia Ehrlich e 25 dipendenti, han no raggiunto un miglioramento delle prestazioni atletiche, aumentando il livello di benessere fisico, migliorando la risposta competitiva, durante l'allenamento e le partite, aiutando nel recupero della capacità muscolare.

Altre lesioni che possono essere prevenute con l'agopuntura sono distorsioni della caviglia, che influenzano i legamenti, innescando molti dolori e limitazioni della funzione articolare. France ${ }^{26}$, utilizzato i punti di ashi (punti dolorosi alla palpazione), associati alla vescica 60 (Figura 2A) per eliminare il dolore; la cistifellea 39 che rinforza le ossa, i tendini e combatte l'algia alle estremità; e cistifellea 40 stimola Qi e xue; stomaco 41 (Figura 2B) che tratta i cambiamenti laterali della caviglia e dello stomaco 36 (Figura 1B) eliminando l'ostruzione e stimolando la circolazione di Qi e xue locale.

Figura 2 A: vescica 60 - tra il tendine d'Achille e il bordo del soleolo laterale della caviglia, al livello del punto più alto del maleolo; B: cistifellea 39 - 3 tsun sopra il punto più alto del maleolo esterno, in depressione tra perone e tendini fibulari lunghi e corti e cistifellea 40 - sul lato antero-inferiore del maleolo esterno, nella depressione laterale del tendine del muscolo estensore digitale lungo ; stomaco 41 - nel punto medio dorsale della caviglia sopra il legamento crociato, tra i tendini del muscolo lungo estensore halux e il lungo estensore delle dita.

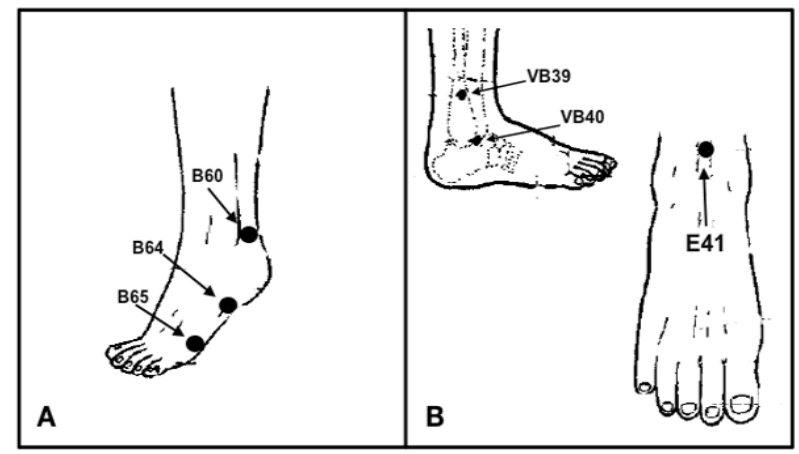

Fonte: Wen ${ }^{17}$ 
Alcuni atleti possono essere sorpresi con distorsione, che è definita come una lesione del tendine cervicale che provoca dolore muscolare e rigidità. C'è un protocollo per porre rimedio a questo problema e restituire l'atleta ai suoi compiti. Wadsworth ${ }^{27}$, utilizzato in aggiunta ai punti ashi che stimolano la circolazione di Qi e xue, la cistifellea 39 (Figura 2B), cistifellea 20 atti nella circolazione di Qi e xue, tendini rilassanti e ammorbidire il dolore; il governatore estrosappare 14 il fattore patogeno e regola il meridiano Qi; vescica 10; intestino tenue 14; cistifellea 21 (Figura 3A) stimolare la circolazione di Qi e xue. Se blocco articolare limita flessione ed estensione, utilizzare il punto della vescica 60 (Figura 2A). Punti intestinali tenue 3, polmone 7 promuovono la circolazione di Qi e xue del collo, se ha limitazioni di rotazione, utilizzare il punto dell'intestino tenue 7 che drena il meridiano Taiyang della mano (Figura 3B). 
Figura $3 \mathrm{~A}$ : cistifellea 20 - al di sotto del bordo occipitale in depressione tra i muscoli trapezioe e sterno-clidomastoide, sul margine dei capelli e della cistifellea 21 - nel punto equidistante tra il Dazhui (VC14) e l'acromion alla spalla, 1 tsun sopra il punto tianiliano; vaso del governatore 14 - nel punto medio tra i processi spinosi; settima vertebra cervicale e prima vertebra dorsale; vescica 10 - al livello tra le spine della seconda e della terza vertebra, 1,3 tsun laterale della linea mediana dorsale, sul lato del bordo del muscolo trapezio e dell'intestino tenue 14 - 3 tsun laterali dell'asse della vertebra; a livello orizzontale del bordo inferiore del processo spinoso della prima vertebra dorsale; B: polmone 7 - sul lato mediale dell'avambraccio, 1,5 tsun sopra la linea del polso tra i tendini del muscolo adduttore del pollice lungo e il muscolo estensore lungo carporadiale; intestino tenue 3 - lato ulnare della mano, dietro l'articolazione metacarpo-falange del quinto dito, tra la pelle chiara e scura; Intestino tenue 7 - 5 tsun sopra il polso, sul lato ulnare del muscolo estensore carpale-ulnare.

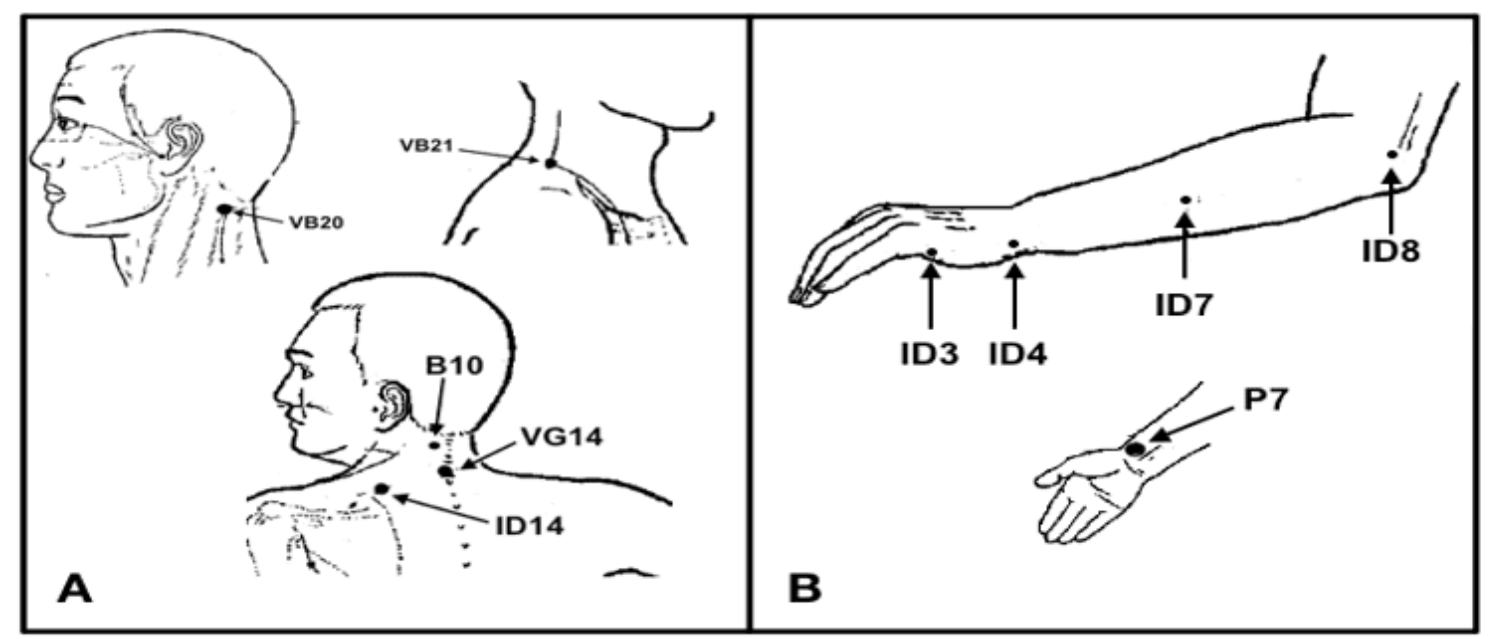

Fonte: Wen ${ }^{17}$

Sovraccaricare l'articolazione del gomito, colpisce, soprattutto i giocatori di tennis, e può portare a un blocco di Qi e xue, innescando un'infiammazione cronica chiamata epicondilite. I punti Ashi sono utilizzati nella regione che promuove la stimolazione Qi. 28

Per il trattamento delle lesioni del polso, come: tendinite o tenosisi del pollice, estensori e flessori delle dita, Francia ${ }^{26}$, ha usato il punto di ashi associato all'intestino crasso 5 
per disperdere il calore del meridiano; intestino tenue 5 eliminando il calore e sbloccando il polso (Figura 4A); Triplo riscaldatore 4 che oltre ad eliminare il calore cessa l'infiammazione e l'intestino crasso 11 (Figura 4B) sbloccare qi e xue calmare il dolore.

Figura $4 \mathrm{~A}$ : intestino tenue 5 - sul lato ulnare del polso, nella depressione tra il pisiforme e il processo stiloide ulnare; Intestino crasso 5 - sul lato posteriore radiale del polso, un po 'distale dell'osso radio, dove c'è depressione tra i tendini del muscolo estensore breve e lungo del pollice, quando si allunga e si apre il pollice. B: triple heater 4 - on the dorsal side of the wrist, in the depression in the middle of the dorsal fold of the wrist, between the tendons of the common digital extensor muscles and digital extensor of the fifth finger;large intestine 11 - on the radial side of the elbow, in the brachioradial muscle; quando si piega il gomito, in depressione radiale alla fine della linea cubitale.

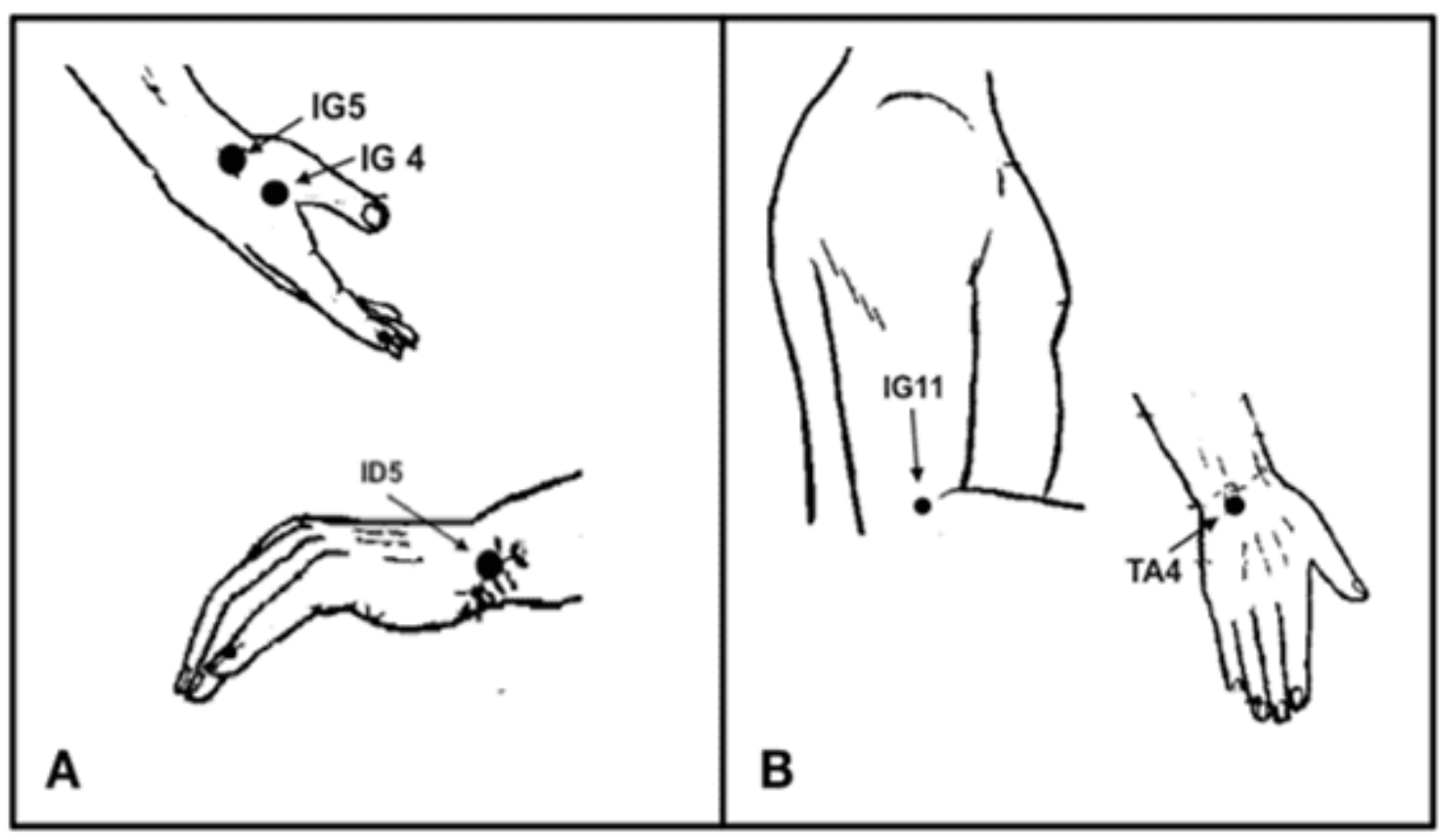

Fonte: Wen ${ }^{17}$

Per il mal di schiena, Barela 17, punti ashi associati con vescica 23 e vescica 25 per stimolare Qi locale e Xue, vescica 40 (Figura 5) che elimina dolore e calore. 
Figura 5: vescica 23 - 1,5 tsun, laterale del bordo inferiore del processo spinale della vertebra (L2); vescica 25 - 1,5 tsun, laterale del bordo inferiore del processo spinale della vertebra (L4); vescica 40 - 3 tsun, laterale dell'asse (Du-Mai), al livello del bordo inferiore nella seconda colonna vertebrale della vertebra (T2).

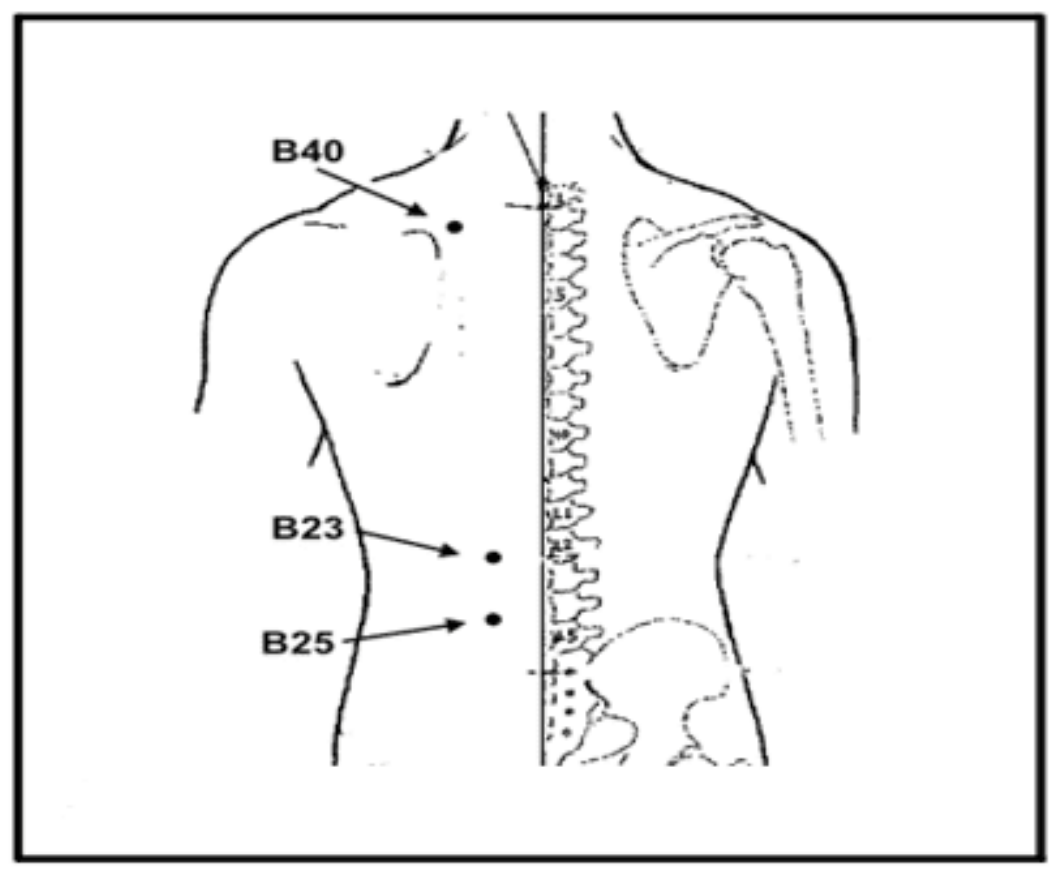

Fonte: Wen ${ }^{17}$

Ora un altro infortunio che preoccupa gli atleti di calcio, sono legamenti, meniscali e tendini del ginocchio. Questo sono trattati con punti ashi, associati con cistifellea 33 (Figura 6) che rilassa tendini e tratta il dolore; vescica 40 (Figura 5) elimina il dolore articolare, edema e calore, stimolando Qi e xue; stomaco 36 (Figura 1B) che regola il flusso di Qi e xue del meridiano eliminando l'ostruzione. ${ }^{29}$ 
Figura 6: cistifellea 33 - sul bordo laterale del ginocchio, 3 tsun sopra il punto di yanglingquan (VB34), nella depressione superiore dell'epitenome laterale del femore.

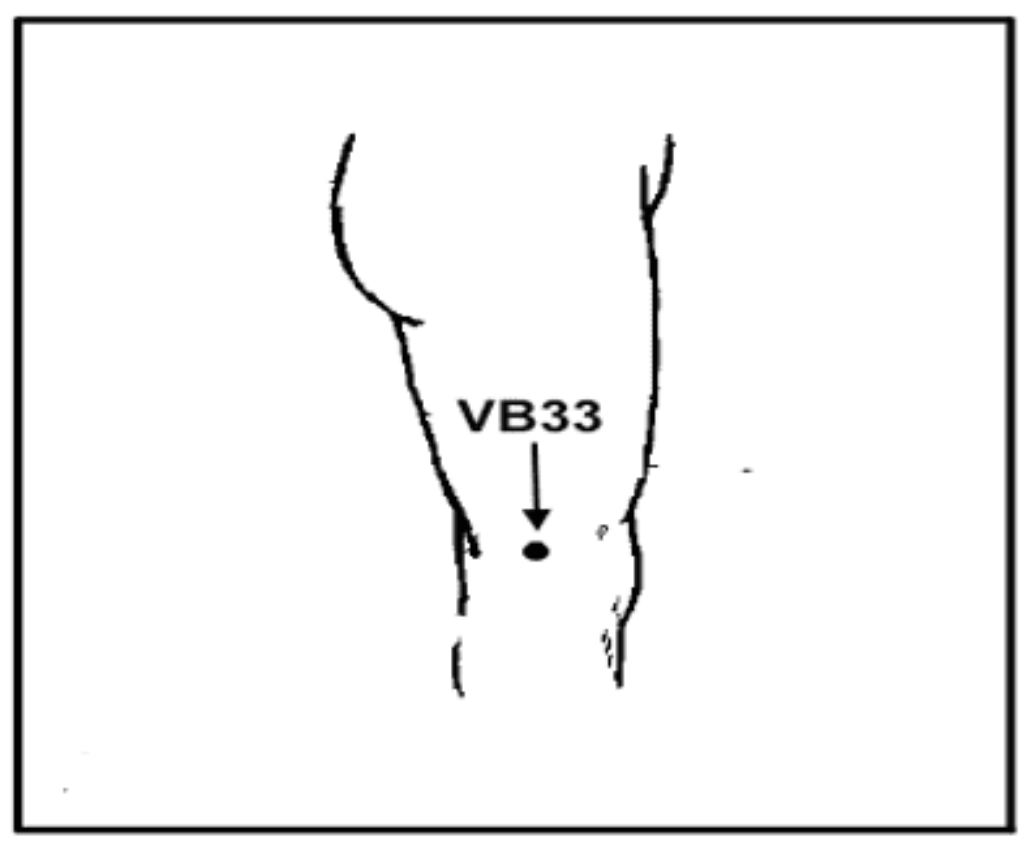

Fonte: Wen ${ }^{17}$

Gemeo ${ }^{30}$, ha dichiarato che l'agopuntura può promuovere un miglioramento della capacità fisica degli atleti, prevenire e trattare gli infortuni che possono verificarsi prima, durante o dopo le competizioni sportive, oltre ad assistere e influenzare fattori emotivi come l'ansia, irritabilità, insonnia e depressione, che può ostacolare direttamente la capacità atletica di questi individui. Ha sviluppato un protocollo specifico utilizzando punti di agopuntura master, selezionando secondo la modalità (Grafico 1):

Tabella 2: Gemeo ${ }^{30}$

\section{Maestro dell'energia vaso governatore 19 (Figura 7) morale}




\begin{tabular}{|c|c|}
\hline $\begin{array}{l}\text { Maestro dell'energia } \\
\text { generale }\end{array}$ & $\begin{array}{l}\text { influenza l'energia generale, agendo sull'energia } \\
\text { ancestrale e postnatale - vaso del governatore } 4,6,10 \text {, } \\
13 \text { (Figura } 7 \text { ) }\end{array}$ \\
\hline $\begin{array}{l}\text { Maestri degli arti } \\
\text { superiori }\end{array}$ & $\begin{array}{l}\text { agisce sui movimenti dell'avambraccio, sui polsi, sulle } \\
\text { mani e sulle spalle: triplo riscaldatore } 15 \text { (Figura } 7 \text { ) }\end{array}$ \\
\hline Maestro dei polmoni & interferisce nel torace anteriore: polmone 1 (Figura 7) \\
\hline Master posteriore & $\begin{array}{l}\text { diaframma, respirazione e nn. frenico: bladder17 (Figura } \\
\text { 7) }\end{array}$ \\
\hline Maestro del ventre & influenza sulla vita addominale: stomaco 27 (Figura 7) \\
\hline Maestro dei reni & Atti di vita lombare: vescica 47 e vescica 52 (Figura 8) \\
\hline Hip master & $\begin{array}{l}\text { agisce il mm. glutei e articolazione coxofemorale (Figura } \\
\text { 8) }\end{array}$ \\
\hline Maestro della coscia & $\begin{array}{l}\text { agisce nella muscolatura delle cosce: cistifellea } 30 \\
\text { (Figura 8) }\end{array}$ \\
\hline $\begin{array}{l}\text { Maestro degli arti } \\
\text { inferiori }\end{array}$ & $\begin{array}{l}\text { influenza l'azione dei piedi e dei muscoli in generale: } \\
\text { vescica } 58 \text { (Figura 8) e stomaco } 36 \text { (Figura 1B) }\end{array}$ \\
\hline $\begin{array}{lrr}\text { Punti per } & \text { migliorare } \\
\text { l'equilibrio } & \text { e } & \text { la } \\
\text { riflessione } & & \end{array}$ & $\begin{array}{l}\text { vaso idea } 4 \text {, vaso conception } 6 \text { (Figura 9), intestino } \\
\text { crasso } 11 \text { (Figura } 4 \mathrm{~B} \text { ), vescica } 46 \text { (Figura } 8 \text { ), vaso } \\
\text { governatore } 4 \text { (Figura } 7 \text { ). }\end{array}$ \\
\hline
\end{tabular}

Figura 7: vaso del governatore 4 - nella linea centrale della colonna vertebrale della colonna vertebrale; nello spazio tra le spine della seconda e della terza vertebra lombare; vaso governatore 6 - nella linea centrale della colonna; tra l'undicesimo e il dodicesimo processo spinoso delle vertebre dorsali; vaso governatore 10 - nella linea centrale della colonna; tra il sesto e il settimo processo spinoso delle vertebre dorsali; vaso del governatore 13 - nella linea centrale della colonna; tra il primo e il secondo processo spinoso delle vertebre dorsali; vaso del governatore $19-1,5$ tsun dietro Baihui (VG 20); stomaco 27 - 2 tsun sotto l'ombel e 2 tsun accanto alla linea mediale sul bordo laterale del muscolo retto-addominale; vescica 17 - 1,5 tsun dell'asse, al livello del bordo inferiore del processo spinale della vertebra (T7); polmone 1 - sul lato anterolaterale del torace, sotto il punto Yunmen (P2) (pit tra la clavicola e la spalla), nello spazio tra la prima e la seconda costola, 6 tsun sul lato intermedio del corpo; 
triplo riscaldatore 15 - nella fossa sopraproforberare, tra Quyuan (ID13) e Jianjing (VB21).

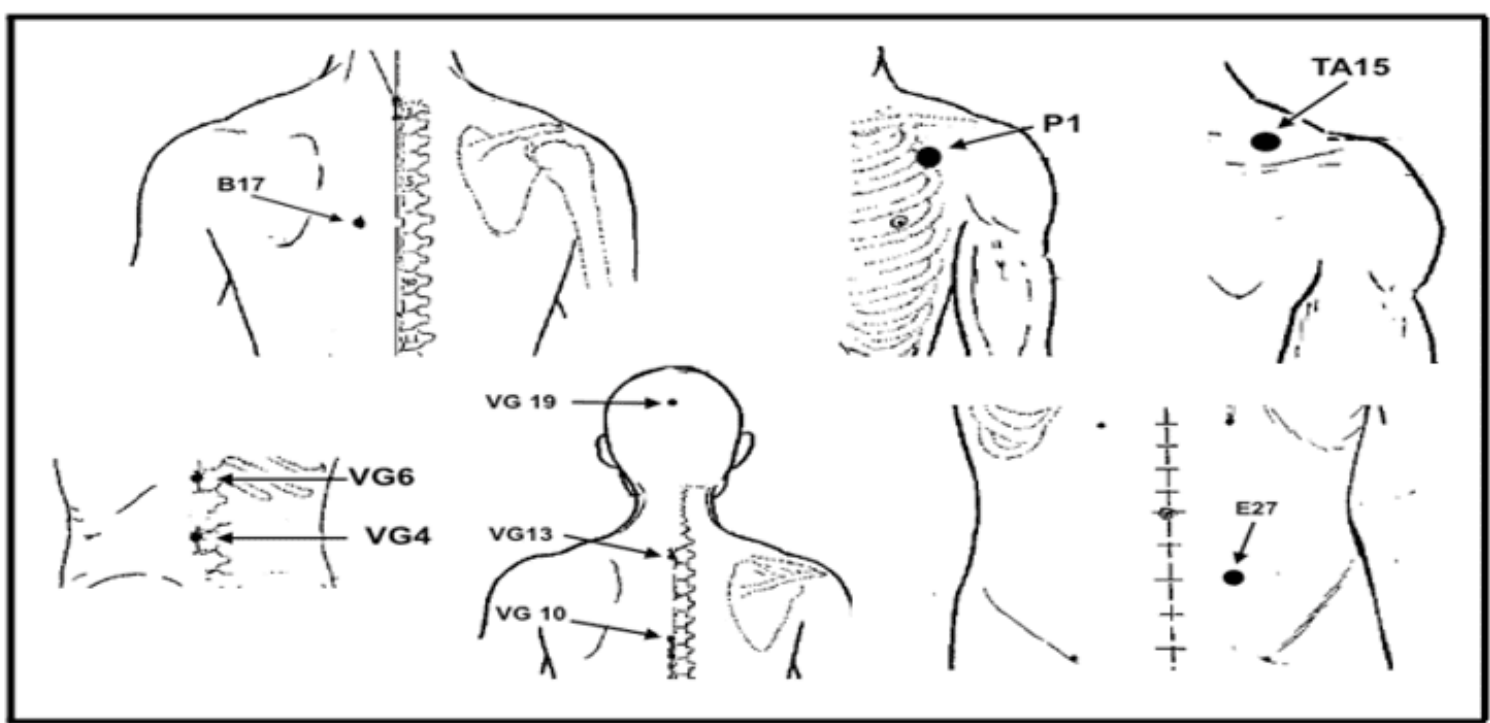

Fonte: Wen ${ }^{17}$ 
Figura 8: vescica 46 - 3 tsun, laterale dell'asse, al livello del bordo inferiore della colonna vertebrale (T9); vescica 47 - 3 tsun, laterale dell'asse, al livello del bordo inferiore della colonna vertebrale vertebrale ( $\mathrm{T} 10)$; vescica 52 - 3 tsun, laterale dell'asse, a livello della seconda colonna vertebrale del sacro, sul bordo laterale dell'articolazione iliossacrale; vescica 58 - I tsun sotto il lato laterale del punto chengshan (B57); 7 tsun sopra il tallone, sul lato laterale del tendine muscolare gastrocnemius; cistifellea 30 - nel gluteo, nella linea tra la pausa sacra e il prominente del trochanter più grande; un terzo della distanza laterale sul bordo inferiore del muscolo piriforme; punto dell'anca extra - al centro della linea che va dall'estremità superiore della scanalatura intergluteale alla parte della cresta esterna dell'ischio.

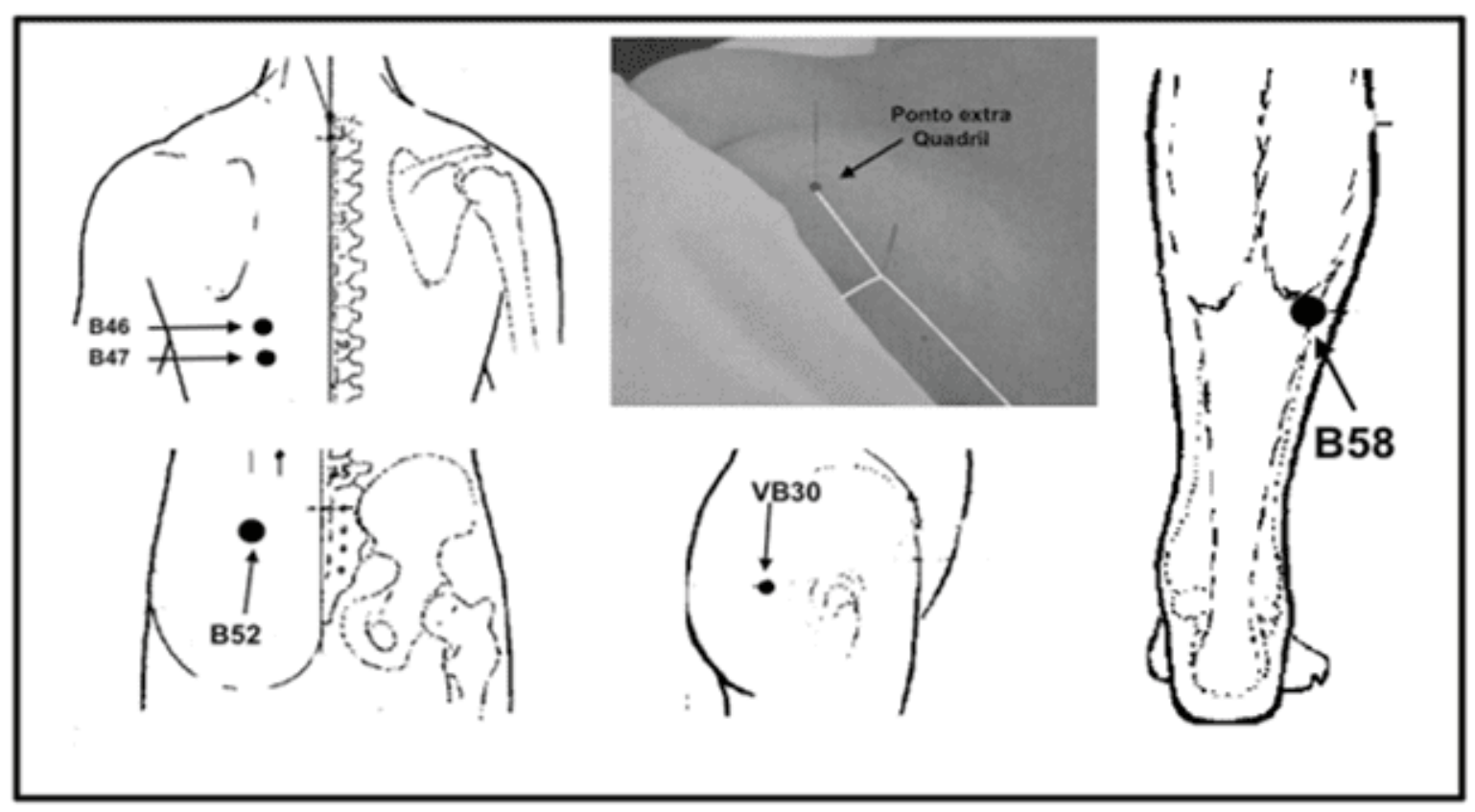

Fonte: Wen ${ }^{17}$; Rosseto ${ }^{31}$ 
Figura 9: concepimento vaso 4 - 3 tsun sotto l'ombelico, nella linea centrale dell'addome; concepimento vaso 6 - 1,5 tsun sotto l'ombelico, nella linea centrale dell'addome.

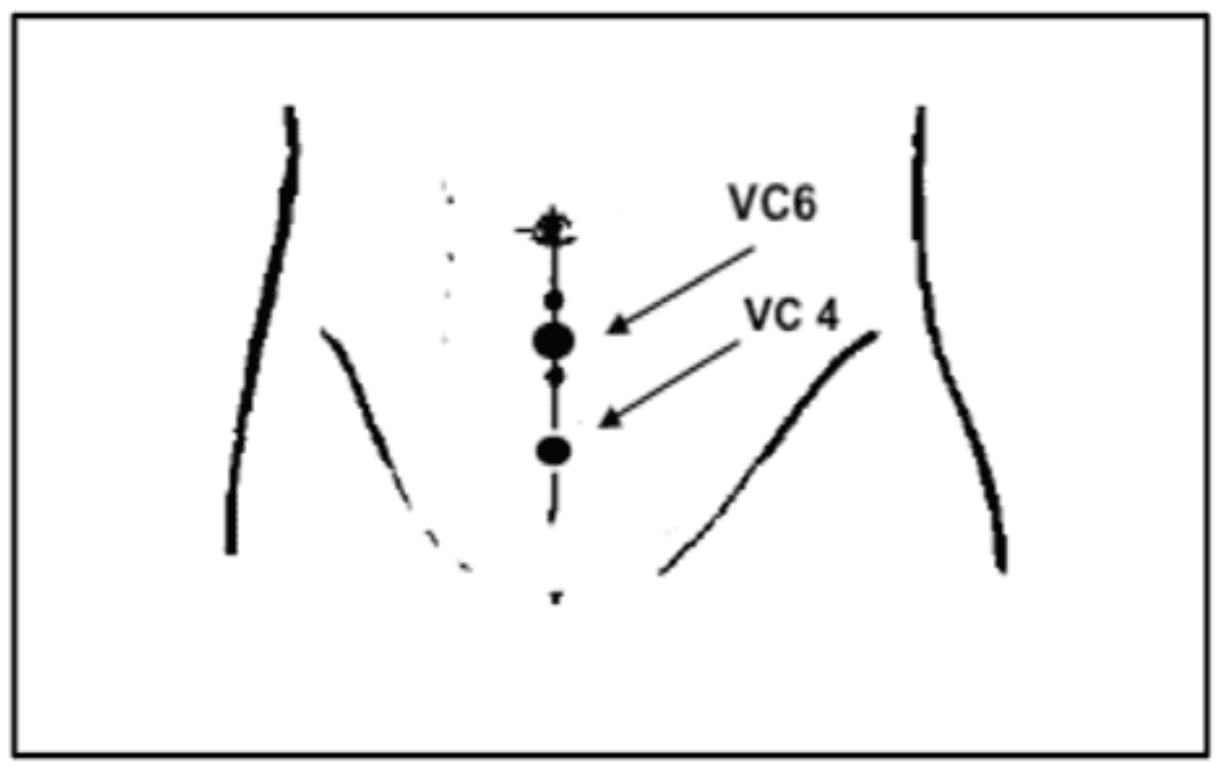

Fonte: Wen ${ }^{17}$

\section{ACUPUNCTURE NEL IMPROVEMENT DI PERFORMANCE ATLETICA}

Quando si tratta di agopuntura nella prevenzione degli infortuni negli atleti, non dobbiamo dimenticare che il raggiungimento di questo obiettivo preventivo, di conseguenza miglioriamo la qualità e l'integrità fisica e possiamo intervenire nel migliorare le prestazioni.

Nei paesi asiatici l'uso dell'agopuntura sviluppa un benessere fisico per gli atleti, regolato da nervosi, endocrini, sistemi immunitari e, di conseguenza, un miglioramento delle condizioni fisiche in diverse modalità. ${ }^{32,31,34}$ Inoltre ci sono punti applicati prima, durante e dopo l'allenamento e le partite, che aiutano nel mantenimento dei muscoli, come nel ridurre l'acido lattico, ridurre il dolore, la fatica, accelerare il recupero e migliorare le prestazioni. ${ }^{35}$

L'agopuntura stimola il rilascio di sostanze biochimiche che allevia l'affaticamento muscolare da esercizi intensi, oltre a promuovere una maggiore attività antiossidante, 
diminuendo i tassi di stress ossidativo. Durante uno studio, gli atleti hanno ricevuto un trattamento con elettroagocupuncture per 30 minuti, per un periodo di 30 giorni e il risultato è stato il significativo aumento di dismutasi superossido (SOD) e diminuzione malondiale del sangue (MDA). La dismutasi superossido è un enzima con effetti antiossidanti che protegge le cellule dalle sostanze tossiche esercitando una potente risposta antinfiammatoria nel corpo. L'MDA è legato alle malattie infiammatorie e degenerative, permettendo ai ricercatori di concludere che l'elettroamcupuntura diminuisce i tassi di MDA e aumenta il SOD, contribuendo al sollievo dalla fatica e migliorando le prestazioni fisiche. ${ }^{36}$

Anche se è una tecnica millenaria, il suo uso migliora le prestazioni fisiche e mentali dell'atleta è recente. Luna, ha pubblicato una ricerca dimostrando che l'agopuntura ha statisticamente migliorato la massima forza e potenza degli atleti velocisti (100, $200 \mathrm{e}$ 400 metri poco profondi senza barriere) e da allora la ricerca a questo proposito è stata approfondita. ${ }^{37}$

In una ricerca, Santos ${ }^{20}$ ha usato atleti Maschi Handbol tra i 15 e i 17 anni, dove ha eseguito due colpi da 100 metri in ogni atleta (preagope). Dopo 45 minuti di applicazione del protocollo scelto per il trattamento, ha rivalutato allo stesso modo. I punti scelti sono stati: vescica 58 (Figura 8) padrone delle gambe e del piede; stomaco 36 (Figura 1B) tonifica la muscolatura striata e viene utilizzata per potenziare il trattamento; punto principale extra dei fianchi (Figura 8) tonifica i glutei e aumenta la flessibilità dell'articolazione coxofemorale; cistifellea 30 (Figura 8) padrone delle cosce e promuove la flessibilità per il movimento degli arti inferiori; polmone 1 (Figura 7) padrone dei polmoni promuove il lavoro respiratorio efficiente; vescica 17 (Figura 7) padrone della schiena, diaframma dorsale shu e punto di influenza di Xue che regola il ritmo respiratorio; triplo riscaldatore 15 (Figura 7) maestro di braccia che comanda il gruppo muscolare articolare di spalle, nuca, colonna vertebrale cervicale, prime sei vertebre toraciche, braccia, avambracci, polsi e mani.

I risultati hanno identificato un miglioramento significativo, con una diminuzione del tempo nella seconda batteria dei test di 100 metri. II miglioramento medio dei tempi è 
stato del $5,72 \%$ e l'atleta con le migliori prestazioni raggiunte ha raggiunto una percentuale del $10,84 \%$.

Questa media del 5,72\% raggiunta nel miglioramento delle prestazioni sembra piccola, ma se confrontata con i risultati di importanti competizioni, possiamo vedere la validità della pratica: ai Giochi panamericani di Rio de Janeiro 2007, nella finale dei 100 metri bassi maschi a differenza tra il primo e l'ottavo posto era 0,23 secondi, rappresentando un tempo del $2,26 \%$ superiore, cioè se questo atleta raggiungesse un miglioramento del $5,72 \%$ nelle sue prestazioni, probabilmente otterrebbe altri risultati.

C'è poca attenzione scientifica, quando trattiamo, degli effetti dell'agopuntura sui miglioramenti nella risposta fisiologica indotta dall'esercizio fisico e pochi diretti agli atleti ad alte prestazioni. ${ }^{24,38} \mathrm{Ma}$ Rossetto ${ }^{31}$ presenta un protocollo per migliorare le prestazioni atletiche nel calcio e nel rugby utilizzando i seguenti punti: vescica $52 \mathrm{e}$ vescica 58 (Figura 8); vescica 17 e polmone 1 (figura 7 ).

Il punto della vescica 58, tonifica la muscolatura striata di tutto il corpo, l'articolazione delle ginocchia, delle caviglie e dei piedi. È un punto importante per evitare la fatica, soprattutto se usato con lo stomaco 36 . La vescica 17 tonifica i muscoli dorsali, i legamenti articolari e aumenta anche la flessibilità delle articolazioni vertebrali stesse, promuovendo un'azione regolatoria sul diaframma, il ritmo respiratorio e i nervi frenici. La vescica 52 tonifica l'intera regione dei muscoli lombari e promuove la forza di volontà. II polmone 1 è usato per rendere efficiente il lavoro respiratorio ed è correlato ai nervi supclavicolari e intercostali (con inserimento più profondo) che assistono nel plesso brachiale. L'uso con il punto della vescica 17 regolarizza il ritmo respiratorio.

Come Bopp Limoge 39, applicato un protocollo fisso a tre punti: fegato 5 (Figura 10), triplo riscaldatore 15 (Figura 7) e vescica 58 (Figura 8), aggiunto ad altri due punti casuali, placebo, in 35 atleti, ottenendo un miglioramento significativo nelle prestazioni atletiche degli individui testati. 
Figura 10: fegato 5 - 5 tsun sopra mediale maleolo; sul bordo posteromediale della tibia.

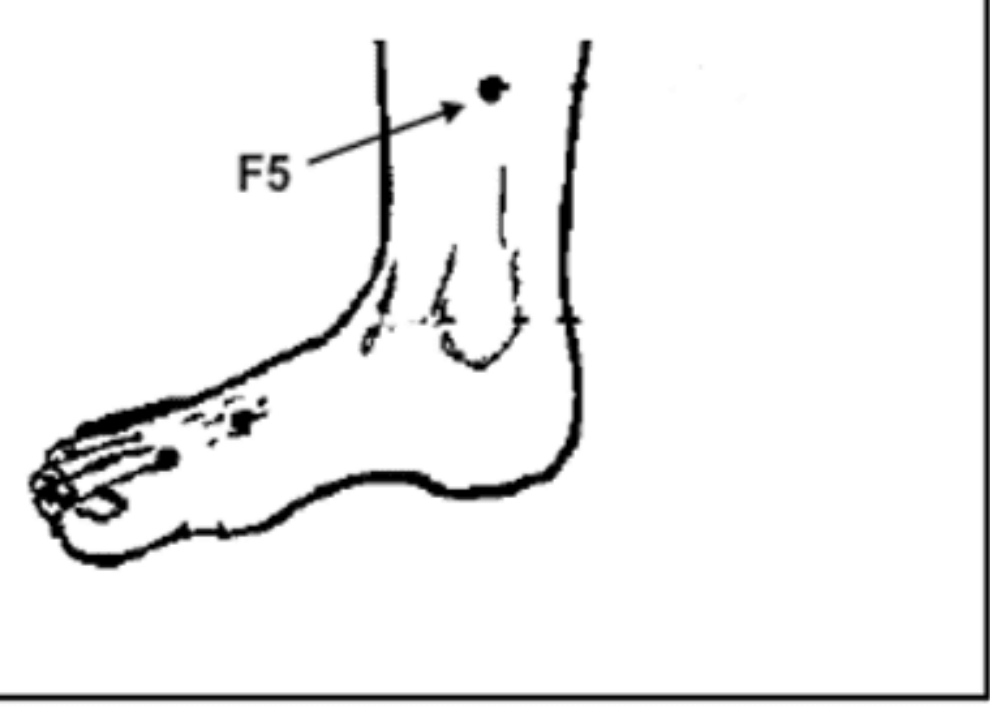

Fonte: Wen ${ }^{17}$

Lo studio di Akimoto ${ }^{38} 8$ corrobora i risultati di diversi studi relativi al miglioramento della capacità fisica e mentale, dove ha testato ventuno atleti di calcio durante la fase di competizione, valutando biochimicamente IgA e cortisolo, per tasso tensione muscolare e affaticamento, con conseguente aumento del cortisolo e igA salivare inferiore, confermando l'efficace miglioramento delle prestazioni atletiche e mentali.

In un'altra ricerca condotta nel trattamento di lesioni sportive con agopuntura cinetica (associazione di agopuntura con kinesioterapia), in atleti di tennis, calcio, pallavolo, ginnastica olimpica, capoeira, balla e jiu-jitsu; pazienti con disturbi locomotori come: tendinite di Achille, mal di schiena, mal di schiena, prarcicolo, tenosisi, sindrome del tunnel carpale, sindrome del pronador, contusione muscolare, gomito del tennis trompazione e gonalgia a causa di lesioni da menisco. Tutti i 31 atleti testati sono tornati all'allenamento, al massimo, fino alla terza sessione: $53 \%$ è tornato dopo la prima sessione; $37 \%$ dopo il secondo; $11 \%$ dopo il terzo, concludendo che questa tecnica ha agito in modo efficiente accelerando in modo efficiente il tempo di recupero 
degli atleti. La spiegazione di questo è che l'agopuntura agisce nell'inibizione del ciclo dello spasmo - dolore, che porta a un blocco afferente segmentale, blocco discendente sopraspinale, attraverso percorsi piramidali e l'attivazione del processo analgesici endogeni. ${ }^{26}$

In un altro studio, le variabili della forza massima, della forza esplosiva, della resistenza anaerobica e della velocità nei corridoi del velocista ad alto rendimento sono state valutate durante un periodo di transizione. II meridiano del fegato era focalizzato: fegato 1 ; fegato 3 ; fegato 8 (Figura 11A), perché le funzioni energetiche di questo organo sono per memorizzare il sangue, controllare la dispersione, drenaggio e determinare le condizioni del tendine e del legamento, oltre a controllare la parte emotiva, perché un deficit energetico può innescare: depressione; irritabilità; insonnia; sogni inquietanti. II primo acupoint forato è stato la vescica 62 , la cistifellea 34 , il rene 3 (Figura 11B), seguito dalla milza del pancreas 3 (Figura 1A), il fegato 1, il fegato 3 , il fegato 8 (Figura 11A), lo stomaco 36 (Figura 1B) e la chiusura con l'intestino tenue 3 (Figura 3B). Punti di fegato sono stati introdotti nell'ottava sessione. I risultati sono stati di positiva impatto, sviluppando un miglioramento delle funzioni studiate. ${ }^{24}$ 
Figura $11 \mathrm{~A}$ : fegato 1 - 0,1 tsun sopra l'angolo laterale del letto ungueale della punta e del fegato 3 - tra il primo e il secondo metatarsale, dietro le articolazioni metatarsalefalangeali; fegato 8 - alla fine del lato mediale della piega popliteale, sul bordo anteromediale dei muscoli semimembraosi e semitedinanti. B: rene 3 - tra il bordo posteriore del maléolus mediale e il tendine d'Achille; cistifellea 34 - I tsun sotto il ginocchio, nella depressione anteriore e inferiore della testa del perone, nella fascia del muscolo lungo peroreo; bexia 62 - 0,5 tsun sotto il maleolo esterno, nella depressione inferiore del maleolo.

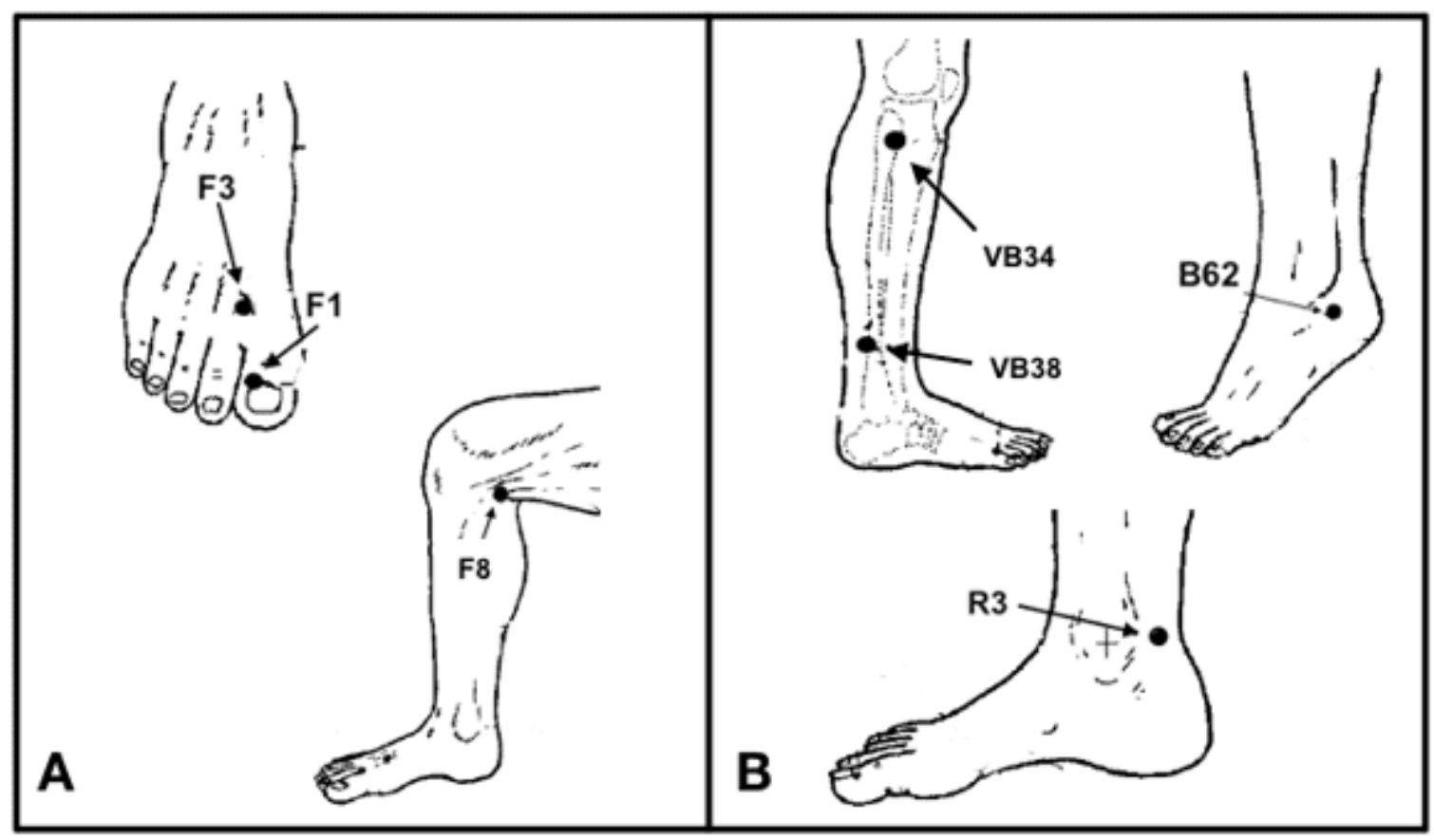

Fonte: Wen ${ }^{17}$

Ehrlich ${ }^{25}$, d'altra parte, divideva 36 individui maschi in tre gruppi: uno trattato con agopuntura sistemica; un altro gruppo di controllo; un altro con agopuntura placebo. È stato ottenuto un miglioramento significativo delle prestazioni fisiche e dei parametri emodinamici. Una sessione si è tenuta a settimana presso il governatore vaso 20 , vaso concetto 15 e fegato 13, vescica 43 (Figura 12), stomaco 36 (Figura 1B) e milza pancreas 6 (Figura $1 \mathrm{~A})$ per 5 settimane. Hanno analizzato che la soglia anaerobica è migliorata del $6,62 \%$ e le prestazioni massime nel $7,15 \%$ nel gruppo che ha ricevuto 
l'agopuntura sistemica, già nel gruppo di controllo e nel placebo, non ci sono state alterazioni.

Figura 12: vaso governatore 20 - sulla linea di testa centrale-verticale; 7 tsun sopra il bordo posteriore dei capelli; 5 tsun dietro il margine anteriore dei capelli; vaso conception 15 - 3 tsun sopra 'hongwan (RM12), nella linea centrale dell'addome e del fegato 13 - sul bordo inferiore del punto finale dell'undicesima costola, sul lato dell'addome; vescica 43 - 3 tsun, laterale dell'asse (Du-Mai), al livello del bordo inferiore della colonna vertebrale (T5).

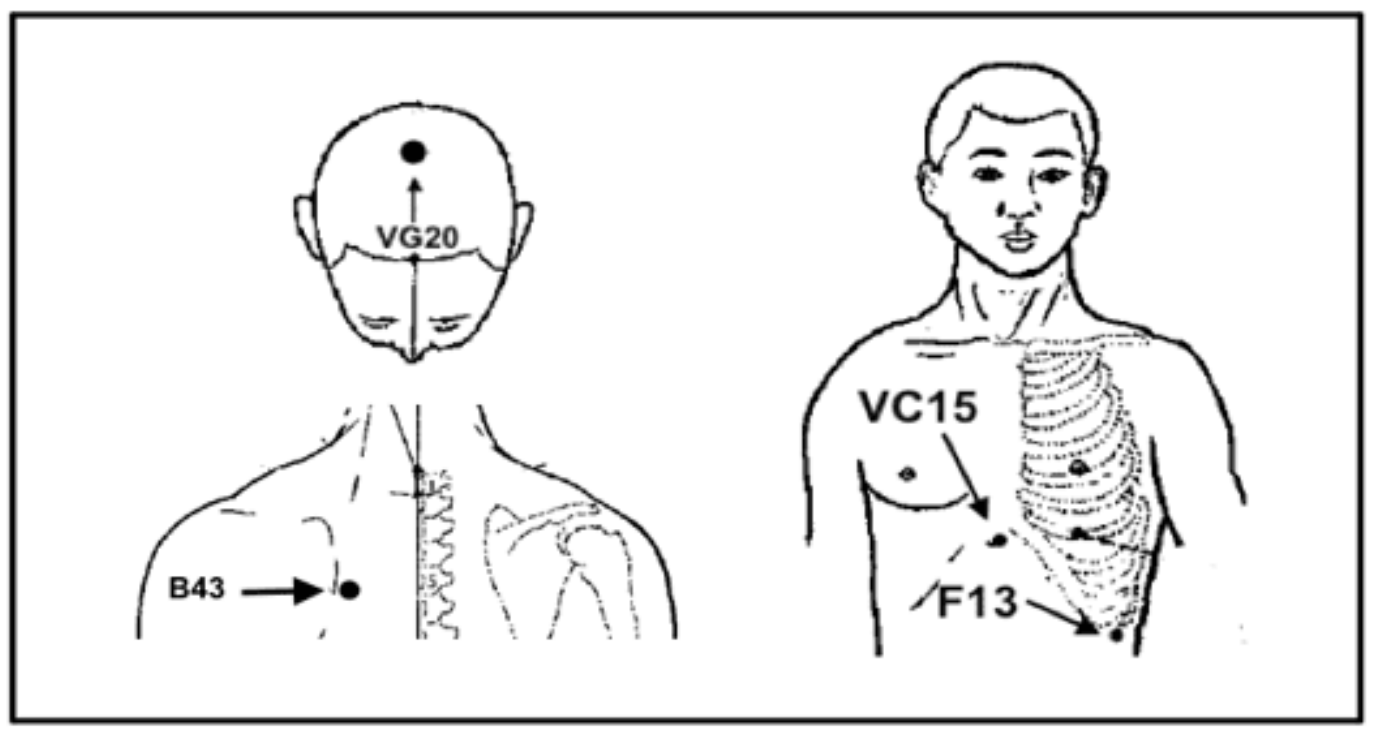

Fonte: Wen ${ }^{17}$

Lin ${ }^{40}$, ha valutato gli effetti dell'agopuntura dell'orecchio sulla frequenza cardiaca (Cmf), il consumo di ossigeno (VO2max) e l'acido lattico del sangue negli atleti di basket d'elite. Sono stati divisi in 2 gruppi di 12 atleti ciascuno, dove: Gruppo 1 agopuntura auricolare; Gruppo 2 - controllo. Ognuno degli atleti ha pedalato su una cyclette fino all'esaurimento, e il Cmax, VO2max e l'acido lattico del sangue sono stati misurati durante il periodo di riposo, dopo il riscaldamento e durante l'esercizio: dopo 5, 30 e 60 minuti. I risultati hanno mostrato che HR e l'acido lattico del sangue erano significativamente inferiori rispetto al gruppo di controllo, dopo 30 e 60 minuti di attività. 
Lo stesso autore, $L^{\text {in }} 41$, in una nuova ricerca, ha valutato le stesse variabili di FCmàx, VO2max e acido lattico del sangue in 30 atleti di basket, suddivisi in 3 gruppi: 1agopuntura sistemica; 2- gruppo farsa; 3-controllo. Nel gruppo 1, sono stati utilizzati il punto di circolazione e la sessualità 6 (Figura 13), stomaco 36 (Figura 1B) e durante lo studio ogni atleta ha eseguito i test su un tapis roulant di esercizio per 15 minuti. Le variabili sono state misurate in riposo e in 5, 30 e 60 minuti dopo l'esercizio, ottenendo una significativa riduzione delle tre variabili dopo 30 e 60 minuti.

Figura 13: circolazione e sessualità 6 - 1 tsun sotto il punto jianshi (PC5); 2 tsun sopra il polso, tra tendini di muscoli palmar lunghi e flessore carpale-radiale

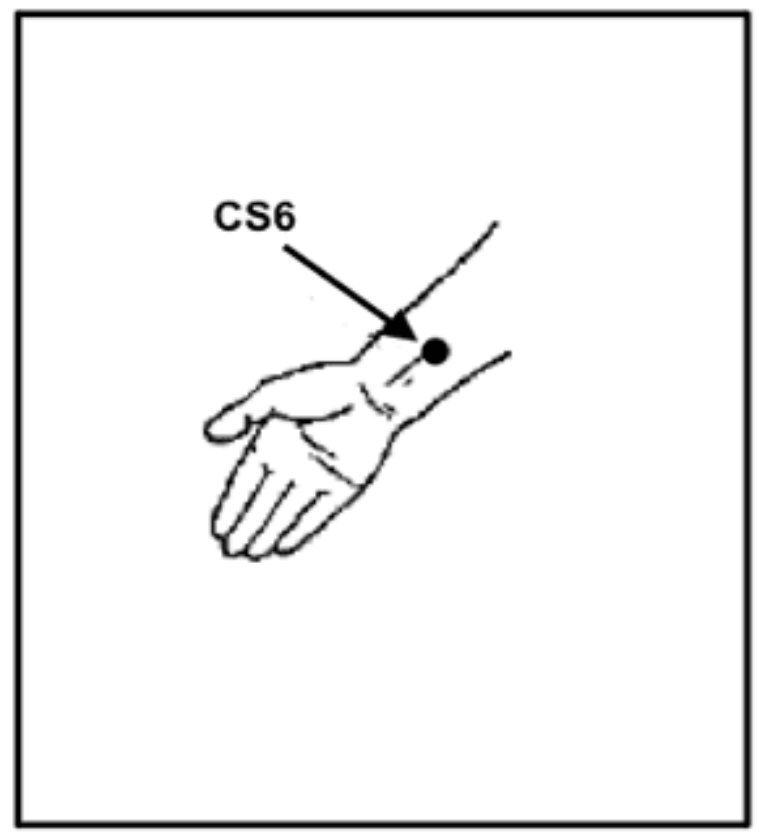

Fonte: Wen ${ }^{17}$

Dhillon ${ }^{42}$, valutato le prestazioni di 20 ciclisti maschi, suddivisi in tre gruppi: trattati con agopuntura sistemica: stomaco 36 (Figura 1B), cistifellea 34 (Figura 11B), fegato 11 (Figura 14), rene 3 (Figura 11B), nave governatore 20 (Figura 12 (Figura 12 ); agopuntura farsa; controllo, senza intervento. Gli atleti hanno pedalato in cyclette per $20 \mathrm{~km}$ alla loro massima velocità supportata. La scala analogica del dolore, visivo, per gli arti inferiori, indotta dall'esercizio; La scala di Borg di sforzo percepito; concentrazioni di lattati nel sangue post-esercizio. II gruppo indotto dall'agopuntura ha 
presentato un punteggio più alto nella Scala Borg, il che significa che il trattamento ha promosso uno sforzo maggiore durante l'attività e ha diminuito il tempo di completamento del test.

Figura 14: fegato 11 - 1 tsun sotto ingano; al bordo antero-mediale della soglia del muscolo adduttore lungo

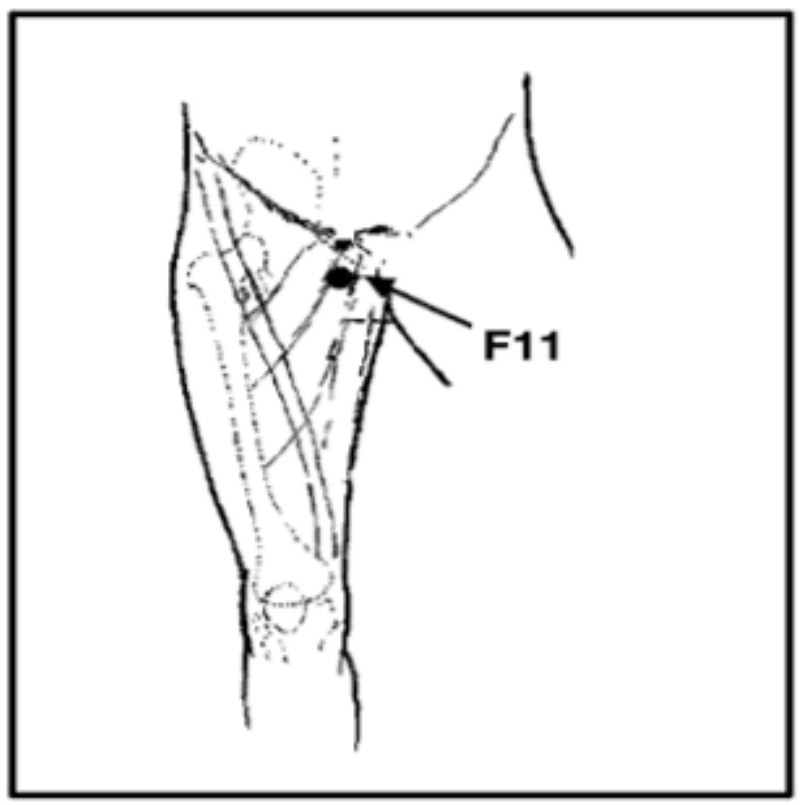

Fonte: Wen ${ }^{17}$

Secondo Hubscher ${ }^{43}$, ha valutato il salto massimo con atterraggio bipodale e forza isometrica massima volontaria dei quadricipiti, con elettromiografia superficiale per misurare l'attività muscolare del retto femorale per 30 secondi di supporto. Trentatré atleti sono stati divisi (13 donne e 20 maschi) in tre gruppi: agopuntura sistemica utilizzando punti di stomaco 36 (Figura 1B), milza pancreas 6 (Figura 1A), concezione vaso 6 (Figura 9); gruppo di agopuntura con punti inesistenti; Gruppo di controllo utilizzato laser disattivato. Come risultato trovato, è stato un aumento non significativo nel salto massimo, ma un importante aumento della forza isometrica dei quadricipiti.

In un'altra indagine $\mathrm{Ge}^{\text {ng }} 44$, ha reclutato 12 uomini sani con studenti di educazione fisica tra i 19 e i 25 anni di età, valutando gli effetti dell'agopuntura dell'orecchio sui 
livelli di acido lattico, indotti dall'esercizio sul tapis roulant $\left(\mathrm{V}^{\mathrm{O}} 2 \mathrm{max}\right)$. Ha scelto i punti: Fegato, Polmone, Subcorteccia, Ghiandole Endocrine e Triple Heater (Figura 15). I risultati sono stati un aumento della capacità di costruzione di ossigeno e diminuzione dei livelli di acido lattico del sangue dopo gli esercizi.

Figura 15: Punti orecchio

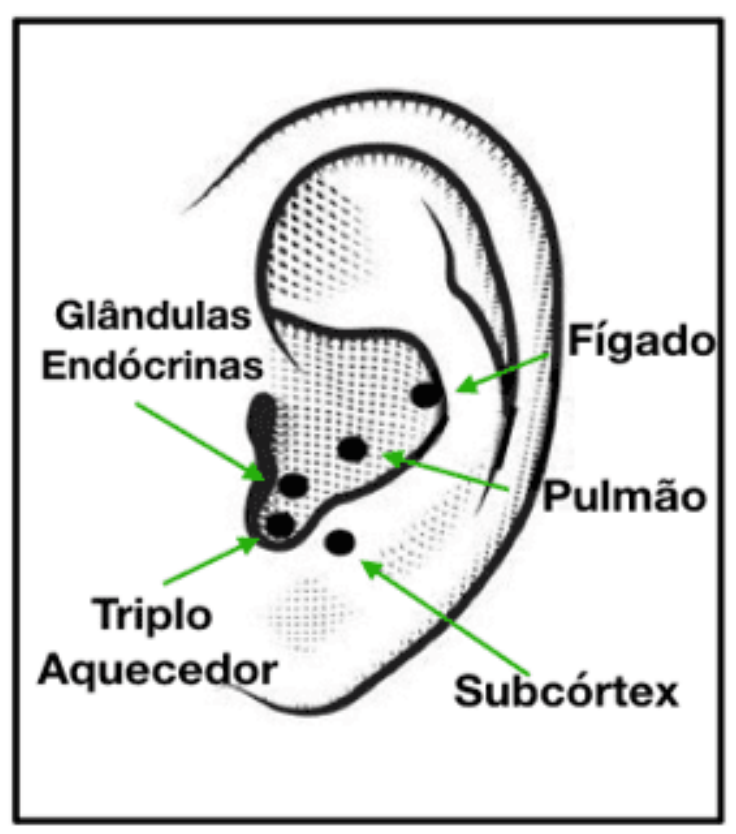

Fonte: Archivio personale dell'autore

Un altro studio di 28 individui maschi divisi in un gruppo di controllo e sperimentale. Una raccolta di dati è stata eseguita prima delle sessioni di agopuntura: Misurazione della frequenza cardiaca basale, attraverso l'ossimetro; misurazione della frequenza cardiaca dopo i test fisici presso la banca di Harvard, con un'altezza di $50 \mathrm{~cm}$, eseguendo salite e discese per 5 minuti; frequenza cardiaca misurata in 1 minuto e mezzo del test, 2 minuti e mezzo e 3 minuti e mezzo; calcolo dell'indice di fitness fisico (IAF); frequenza cardiaca media e il numero di salite e discese durante i test bancari di Harvard. I punti sono stati utilizzati: polmone 10 e vaso conception 17 (Figura 16), intestino tenue 3 (Figura 3B), al fine di tonificare e portare al rilassamento muscolare, rafforzamento dei tendini, colonna lombare e arti inferiori. Nei punti della cistifellea 34 (Figura 11B), i pancreas di milza 4 e il rene 7 (Figura 16) erano correlati alla sedazione 
per potenziare il movimento della circolazione sanguigna (Xue) e della linfa (Jing Yie). Sono state eseguite otto sessioni, una alla settimana e i risultati non hanno mostrato un miglioramento significativo in relazione alla frequenza cardiaca e alla forma fisica. Nell'aumentare le prestazioni fisiche, le salite e le discese dalla Banca di Harvard, i valori erano significativi per il gruppo sperimentale in relazione al controllo. ${ }^{36}$

Figura 16: polmone 10 - sul lato palmar, sopra l'articolazione del primo metacarpo digitale, tra pelli scure e chiare; concepimento vaso 17 - nella linea mediana dello sterno a livello del capezzolo; rene 7 - 2 tsun sopra Taixi (R3); sul bordo antero-mediale dell'unico muscolo; milza pancreas 4 - sul lato mediale del piede; 1 tsun dietro l'articolazione metatarsophal, alla giunzione della pelle scura e chiara.
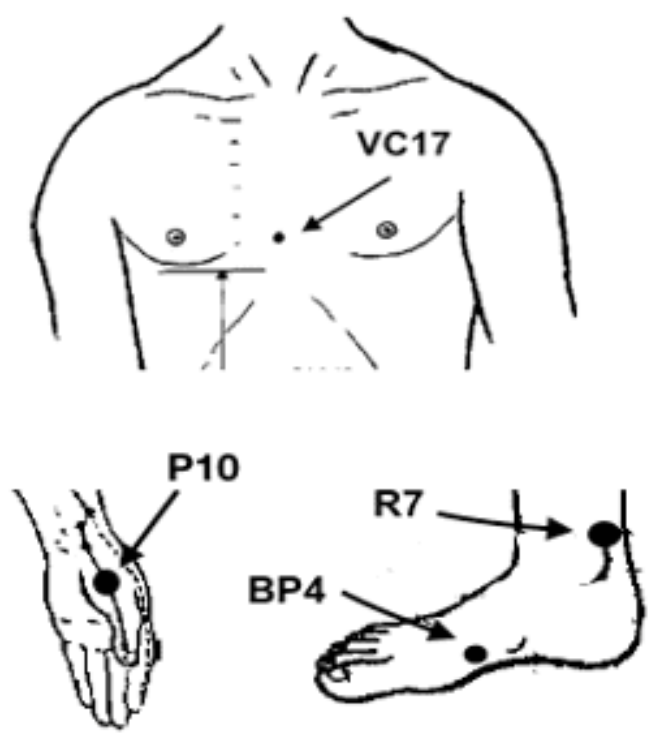

Fonte: Wen ${ }^{17}$

Fonseca ${ }^{45}$, d'altra parte, ha usato un campione con 40 individui maschi che praticano lo sforzo muscolare due gruppi: controllo e intervento per valutare gli effetti dell'agopuntura sulla resistenza muscolare localizzata $(R M L)$ degli arti superiori. II gruppo di controllo è stato sottoposto al test RML per 1 minuto, passando attraverso un periodo di riposo di 30 minuti e dopo, il test è stato ripetuto. 
Nel gruppo di intervento è stata applicata la prova: la prova; riposato per 5 minuti; applicazione dell'agopuntura, tonificando i punti: pancreas di milza 3 (Figura 1A); rene 7 (Figura 16); stomaco 36 (Figura 1B); vaso del governatore 4; riscaldatore triplo 15 (Figura 7); per 25 minuti. Dopo l'applicabilità, il test RML ripetuto, con conseguente miglioramento significativo delle prestazioni muscolari del gruppo di intervento.

Nel 2015, Moniz, ha usato 12 atleti di rugby dove sono stati distribuiti nel gruppo di controllo e nel gruppo di test, eseguendo l'agopuntura per 20 minuti nei punti: cuore 5, vescica 15, vescica 44 (Figura 17) e circolazione e sessualità 6 (Figura 13). Nella seconda sessione settimanale, le forature sono state eseguite nei punti di stress e nei punti di innesco. I risultati hanno suggerito chiaramente che l'applicazione dell'agopuntura porta vantaggi agli atleti rispetto ai problemi muscolari, con il recupero totale a livello di dolore e la limitazione dei movimenti prima del successivo gioco dell'infortunio.

Figura 17: cuore 5 - sul lato ventrale e ulnare dell'avambraccio, sul lato ulnare del tendine del muscolo flessore flessore carpale ulnare; 1 tsun sopra la maniglia; vescica 15 - al livello del bordo inferiore del processo spinale della vertebra (T5), sulla stessa linea verticale del punto Feishu (B13); vescica 44 - 3 tsun, laterale dell'asse, al livello del bordo inferiore della colonna vertebrale vertebrale (T6).

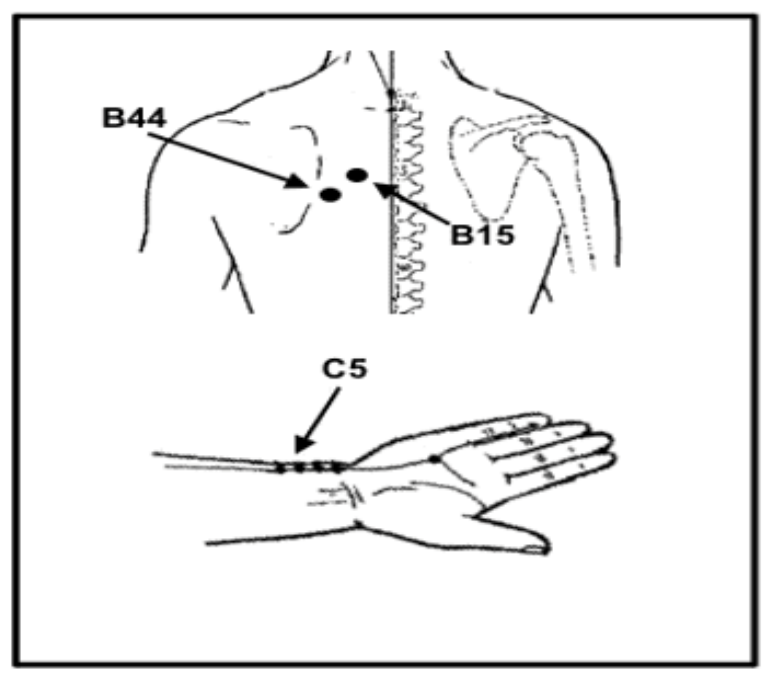

Fonte: Wen ${ }^{17}$ 
La maggior parte degli studi presenta risultati significativi per quanto riguarda il miglioramento delle prestazioni fisiche, portando di conseguenza ad un miglioramento nel tempo di fine dei test. Questo provoca individui sottoposti al trattamento dell'agopuntura, ricevendo un'attivazione della neurofisiologia del controllo del dolore, innescando un miglioramento delle prestazioni muscolari, influenzando notevolmente il risultato finale. ${ }^{46}$

\section{ACUPUNCTURE USATO NEL TREATMENT Di LESIONS}

L'agopuntura agisce in questo processo di cura della malattia, eliminandone la causa, e quindi viene somministrata sia per il trattamento di vari dolori acuti che cronici. Può essere utilizzato nelle procedure chirurgiche, nel miglioramento delle condizioni fisiche e nella prevenzione delle lesioni in vari sport. ${ }^{24}$

Attualmente gli atleti e i loro allenatori cercano il miglioramento delle prestazioni fisiche e della prevenzione attraverso l'allenamento esaustivo, il monitoraggio nutrizionale, la prevenzione fisiotermica, i piani di allenamento, il monitoraggio psicologico e l'uso di nuovi attrezzature sportive per il raggiungimento degli obiettivi. Anche con questi sistemi avanzati, molti atleti preferiscono e si muovono sul percorso più veloce, utilizzando il trattamento illecito, come l'uso di anabolizzanti e droghe. ${ }^{32}$ Secondo Frasca 35, egli afferma che l'agopuntura funziona nell'essere umano nel suo complesso, trattando il dolore, migliorando le prestazioni e agendo direttamente nel sistema psicologico degli atleti generando un aumento della fiducia psichica dello sportivo.

Secondo Santos e collaboratori $2^{0}$, la maggior parte degli atleti hanno avuto l'esperienza di sentire dolore muscolare ad esordio tardivo, che appaiono nei periodi di allenamento, dove l'intensità e il volume del lavoro sono più alti. Un altro tipo di dolore è causato da un infortunio, allontanando gli atleti dall'allenamento e dalle competizioni per lunghi periodi. ${ }^{21}$

Basato sulla neurofisiologia, istoanatomica e neurochimica, il CTM spiega che punteggiando il punto che si trova nei canali dei meridiani specifici, faciliterà il flusso 
di energia in queste strutture sovraccariche da modalità sportive, raggiungere obiettivi analgesici. ${ }^{25,47}$

Altri autori riferiscono che l'applicazione della tecnica ha l'azione di oppioidi endogeni, si verifica il rilascio di z-endorphin identificato nella modulazione del dolore e nell'inibizione della trasmissione nocicettiva a tutti i livelli del sistema nervoso.) attraverso l'uso dell'elettroambronpunto, si verifica una regolazione positiva dell'espressione del rapporto di sintesi dell'ossido nitrico neurale (nNOS) / NADPH diaphorase (NADPHd). Ci sono anche alcuni dati che suggeriscono che oltre agli oppioidi, altri meccanismi sono coinvolti nel trattamento del dolore. $6,48,49,50,51$

La presenza di NO favorisce il miglioramento della vascolarizzazione locale, indicando un'azione omeostotica dell'agopuntura, ma l'aumento di NO nel fascicolo ghiacciato può chiarire questa azione regolatoria dei riflessi somatici causati dalla tecnica. ${ }^{6,52}$ Altri studi osservano il rilascio di serotonina dopo la stimolazione dell'ago. ${ }^{51,52,53}$

L'agopuntura viene applicata al tono, rilassare i muscoli degli atleti e mantenere l'equilibrio energetico del corpo. Oltre all'effetto analgesico, è possibile stimolare altri fattori biologici, attivando l'ipotalamo e la ghiandola pituitaria raggiungendo gli effetti sistemici, come: aumento neuro-ormoni e neurotrasmettitori.2 migliorare le prestazioni dell'atleta nelle rispettive modalità, quindi controllate da sistemi, nervosi, endocrini e immuni. $36,54,55,56,57,58,59,60$ Molti studi hanno dimostrato che l'agopuntura opera in questi tre sistemi, fornendo: sollievo delle tensioni e del dolore muscolare; aumento del flusso sanguigno locale; rilascio di oppioidi endogeni, diminuendo la percezione del dolore; miglioramento della modulazione dell'equilibrio del sistema nervoso simpatico e parasimpatico; modulazione del sistema immunitario; attivazione del tessuto connettivo e dei muscoli rilasciando miosine che hanno effetti antinfiammatori, rilasciando sostanze antiossidanti, diminuendo lo stress ossidativo; sostanze che hanno probabili effetti ergogenici. 5, 13, 61, 62, 63, 64

In uno studio di laboratorio con ratti, Lee e collaboratori, $65 \mathrm{~h}^{\mathrm{a}}$ riferito che l'animale quando sottoposto all'agopuntura e all'esercizio ha presentato una soppressione degli effetti su 5 HT (5 idrossitryptamine) e l'espressione di THP (hydroxylase tryptophan) 
nella rafe dorsale cavie, raggiungendo l'effetto ergogenico, dal momento che il 5 HT impedisce l'esecuzione di esercizio in esseri umani e ratti.

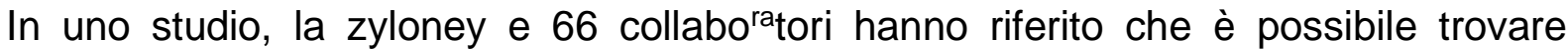
un'attività diversa nel cervello, attraverso la valutazione della risonanza magnetica, durante la procedura dell'ago. Questi cambiamenti appaiono in regioni come: materia grigia epiaquedurale, ipotalamo, corteccia somatosensoriale primaria, giro temporale superiore, corteccia cingolata anteriore rostrale e regione mediale della corteccia occipitale. ${ }^{48}$

Nella sua ricerca, Minori ${ }^{6} 7$ trattata lesioni da stress ripetitivo della spalla, con la combinazione di punti meravigliosi recipiente: triplo riscaldatore 5 - cistifellea 41 (Figura 18) che vengono utilizzati per il dolore cronico e l'intestino tenue 3 (Figura 3B) - vescica 62 ( Figura 11B) per il dolore acuto.

Figura 18: triplo riscaldatore 5 - 2 tun sopra la piega dorsale del polso, tra i tendini del muscolo estensore digitale comune e il muscolo della quinta cifra stessa; cistifellea 41 - in depressione tra il quarto e il quinto metatarso.

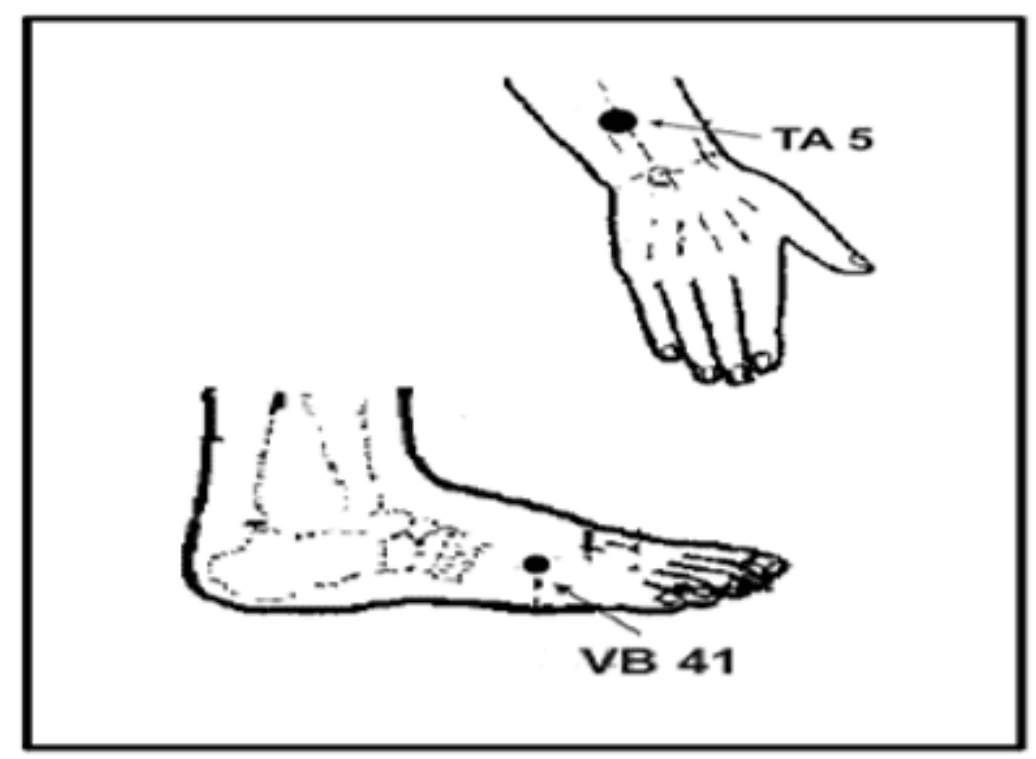

Fonte: Wen ${ }^{17}$ 
Fonte: Wen 17

I punti locali per il trattamento sono: intestino crasso 5 per il dolore al polso; intestino crasso 4 allevia il dolore e gli edemi (Figura 4 A); mal di braccio dell'intestino tenue; triplo riscaldatore 14 indicato per il dolore e l'infiammazione della spalla; intestino crasso 14 (Figura 19) utilizzato per rigidità, paresi, mobilità ridotta della spalla. Punti distanti, dolore alla spalla superiore e anteriore: stomaco 36 per dissipare fattori patogeni esterni; stomaco 37 indicato per il dolore, sensibilità e disturbi della circolazione; stomaco 41 elimina l'umidità, ristagno, vento e lenisce lo shen (mente); lo stomaco 38 elimina il dolore acuto e i disturbi delle articolazioni della spalla (Figura 1B). Per il dolore alla spalla posteriore e superiore: cistifellea 34 indicato per mobilità ridotta, spasmi, disturbi della circolazione e disturbi funzionali di muscoli e tendini; cistifellea 38 disperde il vento e sprigiona calore, attiva il meridiano e i suoi vasi (Figura 11B). Scapola del dolore, d'altra parte: vescica 65 pacifis e rafforza lo spirito, rilascia calore e allevia il dolore; vescica 64 sopprime gli spasmi e rilassa i tendini; vescica 60 allevia il dolore e attiva i punti meridiani ed emotivi (Figura 2A); vescica 18 promuove la calma generale ed elimina gli spasmi (Figura 19) e fegato 8 rilassa tendini (Figura $11 \mathrm{~A})$. 
Figura 19: intestino tenue 9 - nella regione post-bassa dell'articolazione della spalla, sul lato postteroinferiore del muscolo rotondo più grande; triplo riscaldatore 14 - in depressione tra l'acroma e il più grande omero tuberculo, sul bordo del tendine infrarosso; intestino crasso 14 - copre il lato laterale e alcuni radiali del braccio, nel punto disfatto del muscolo deltoide, 3 tsun sotto il punto jianyu; vescica 18 - sulla stessa linea verticale come geshu (B17), al livello del bordo inferiore del processo spinale della vertebra (T9).

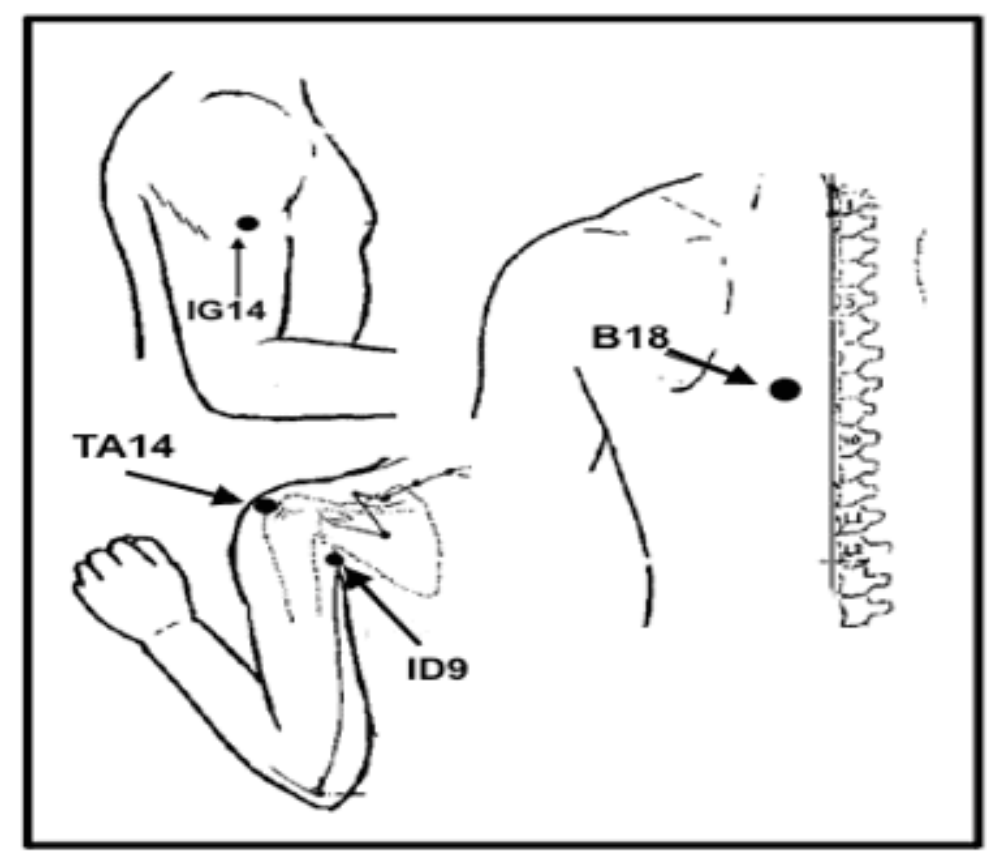

Fonte: Wen ${ }^{17}$

Brum 68, d'altra parte, associato nel suo studio per il trattamento della sindrome piriforme, massaggi e tecniche di agopuntura nei corridoi, al fine di migliorare le funzioni muscolari, flessibilità e sollievo dei sintomi. La ricerca ha incluso atleti di entrambi i sessi di età compresa tra 30 e 60 anni. La sessione si è concentrata sul massaggio (Shiatsu) all'inizio per rilasciare i punti di stress della regione gluteo e coscia sul lato interessato, seguito dall'aghidatura, come segue: in senso orario; antitempo; approfondimento; superficializzare l'ago nelle seguenti regioni: origine e inserimento del muscolo piriforme (faccia pelvica del sacro e trocantro più grande del 
femore); origine del gluteo medio (faccia laterale dell'osso iliaco); limite superiore del gluteo minimo; limite superiore del massimo gluteo; origine dei muscoli del cordino (tuberosità sciaticica); bicipiti femorale muscolo e diafisi muscolare semitedinosa, finito con massaggio.

I risultati hanno mostrato un miglioramento significativo, quando correlati al miglioramento del dolore, flessibilità, palpazione del dolore nel muscolo piriforme e test per la sindrome piriformatra. Questo dimostra l'accordo con lo studio di Hongwen ${ }^{69}$, dove l'efficacia dell'agopuntura è stata del 97,33\% e con la ricerca di France 26 che ha associato gli aghi alla chinesioterapia raggiungendo un sollievo dei sintomi, la potenzializzazione del sistema neuromuscolare, offrendo un ritorno più rapido alle attività sportive.

Nel suo lavoro, Rocha 70, ha verificato il miglioramento dell'attivazione del muscolo retto femorale, attraverso l'elettromiografia, dopo la stimolazione del punto di agopuntura dello stomaco 45 (Figura 20). Un totale di 10 partecipanti (5 maschi e 5 femmine) sono stati selezionati, dove hanno eseguito un esercizio di 2 ripetizioni squat, dove hanno selezionato l'indice di attivazione muscolare più alto tra i due movimenti dal segnale elettromiografico. Poi è stata applicata l'agopuntura, tonificando lo stomaco punto 45 per 10 minuti e poi lo squat isometrico è stato ripetuto nella fase inferiore. Secondo i risultati trovati agopuntura alterato l'attività elettrica del muscolo retto femorale durante la fase isometrica nella fase inferiore dello squat, dimostrando efficace. 
Figura 20: stomaco 45 - 0,1 tsun sul lato dell'angolo laterale del letto ungueale del secondo dito

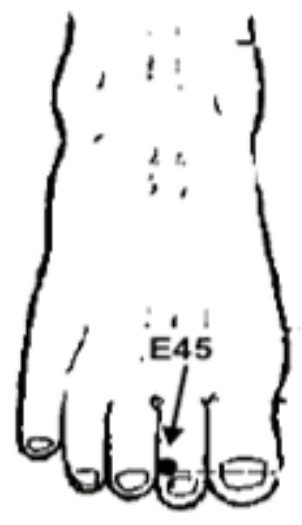

Fonte: Wen ${ }^{17}$

In un altro studio Pinheiro ${ }^{71}$, caratterizzato e depositato alcuni punti per il trattamento della lesione meniscale: fegato 8 (Figura 11A) utilizzato per tonificare il canale epatico con funzione di armonizzazione e tonificazione fegato e sangue $\mathrm{Qi}$, promuovendo la pienezza dei reni e che porta al rilassamento di tendini e muscoli, rafforzando l'Iq del ginocchio. II punto della vescica 40 (Figura 5) si trova nella fossa popliteale abilitando: dispersione di calore e vento perverso degli arti; rilassamento dei muscoli e dei tendini; distruggere i vasi sanguigni; riduce il calore ed elimina la stasi del sangue; diminuzione del regidez e rafforza il ginocchio e lombare. D'altra parte, il punto renale 10 (Figura 21) mobilita l'acqua; toni yin Qi; espelle l'umidità e il calore perverso, trattando il dolore al ginocchio, l'edema delle gambe e il ginocchio intorpidito e il cessum popliteale. 
Figura 21: rene 10 - sul lato mediale della piega popliteale, tra muscoli semitedino e semimembrnous

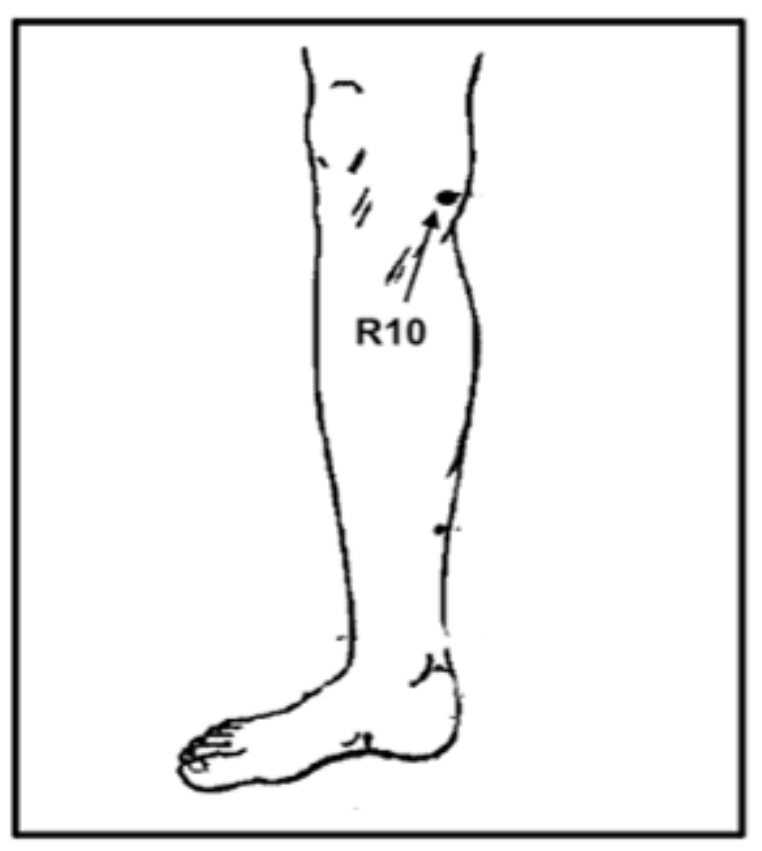

Fonte: Wen ${ }^{17}$

In uno studio sulle disfunzioni della spalla, Nunes ${ }^{72}$, ha esaminato la bibliografia e ha depositato i punti di vasi meravigliosi: intestino tenue 3 (Figura 3B) - vescica 62 (Figura 11B) per bilanciare le funzioni, rilassando muscoli e tendini; i punti del triplo riscaldatore 5 - cistifellea 41 (Figura 18) alleviare il dolore e rilassare i tendini; Punti Ashi; fegato 8 (Figura 11A) e rene 7 (Figura 16) per rafforzare i tendini; stomaco 36 (Figura 1B) eliminando il freddo e l'umidità dalle articolazioni; intestino 4 (Figura 4A) e intestino crasso 15 (Figura 22) che agiscono nel movimento e dolore della flessione della spalla; riscaldatore triplo 14 (Figura 19) e intestino crasso 16 (Figura 22) migliorando la mobilità estensione e il dolore. 
Figura 22: intestino crasso 15 - sulla parte superiore della spalla, sul bordo laterale dell'acromion, ci sono due depressioni, questo punto è nella depressione anteriore; intestino crasso 16 - in depressione tra il bordo superiore e posteriore dell'articolazione acromioclavicolare e la colonna vertebrale della scapola.

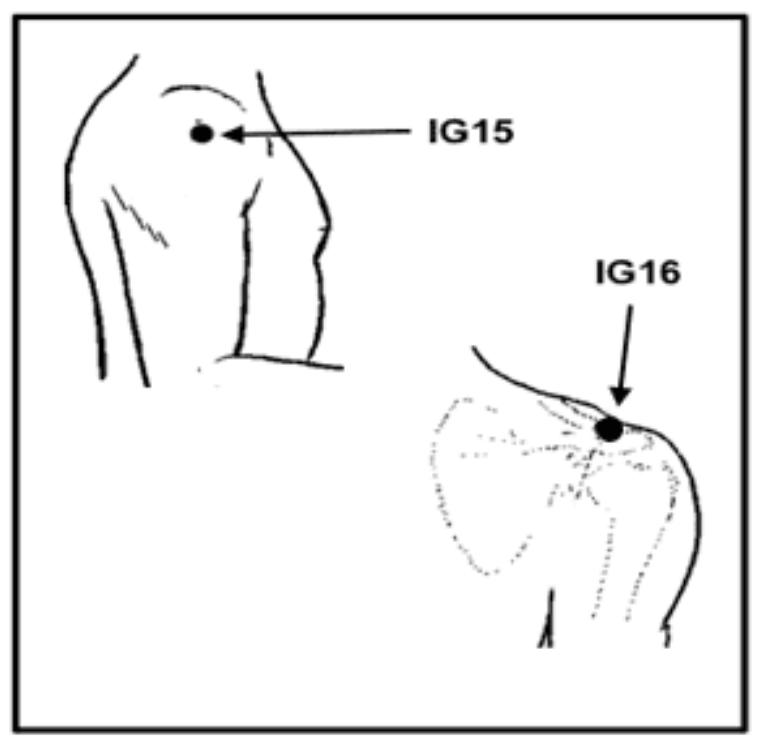

Fonte: Wen ${ }^{1}$

Al fine di verificare gli effetti immediati dell'agopuntura sul dolore muscolare a esordio tardivo (DMIT) e sulla capacità funzionale contrattuale dei muscoli, Parisoto 73 , ha selezionato 30 soggetti (12 uomini e 18 donne), di età compresa tra 20 e 30 anni, praticanti di attività da 2 a 3 volte a settimana. Sono stati divisi in tre gruppi: trattati con agopuntura; tossia placebo; Controllo. Sono stati valutati: soglia del dolore con l'algelometro; picco massimo di forza muscolare isometrica; attività elettromiografica; percezione soggettiva del dolore attraverso la scala analogica visiva.

Dopo le valutazioni, i volontari sono stati sottoposti all'induzione di DMIT, eseguendo la flessione e l'estensione del gomito con carico (filo bicipite), fino al limite massimo di esecuzione del movimento: 3 serie sono state eseguite fino all'esaurimento muscolare con un intervallo di 4-5 minuti. Dopo questa procedura, lo stato in cui il muscolo era prima dell'agopuntura è stato rivalutato. 
I punti scelti per il trattamento del gruppo di agopuntura erano: intestino crasso 4 (Figura 4A), intestino crasso 11 (Figura 4B), stomaco 36 (Figura 1B), cistifellea 34 (Figura 11B), che sono punti analgesici e tonificanti di muscoli e tendini. Nel gruppo farsa, sono stati utilizzati punti placebo e gli aghi sono stati introdotti superficialmente; gruppo di controllo che gli individui si stabiliscono. Entrambi i gruppi rimasero a riposo per 20 minuti.

I risultati hanno indicato: la percezione del dolore con la scala analogica è stata ridotta nel gruppo dell'agopuntura; la soglia di dolore da parte dell'algelometro è aumentata dopo l'intervento in questo gruppo. Dopo l'intervento dell'agopuntura, l'attivazione muscolare dei bicipiti è stata ridotta del $30 \%$ quando è correlata alla finzione e al gruppo di controllo. La forza muscolare, dopo DMIT, è diminuita del $48 \%$ nel gruppo testato, essendo inferiore rispetto alla farsa e ai gruppi di controllo (rispettivamente $58 \%$ e $56 \%$. Tutti i gruppi hanno presentato il massimo deficit di forza muscolare isometrica dopo il protocollo di induzione dmit: l'agopuntura è diminuita del $26 \%$; farsa $29 \%$; controllo $33,5 \%$.

Tuttavia, si è concluso che l'agopuntura è stata efficace nel miglioramento immediato della percezione del dolore e nell'aumento della soglia da parte dell'algelomero, fornendo alterazioni neuromuscolari, ma non migliorando le prestazioni contrattili della muscolatura.

In un altro studio di Tolentin ${ }^{74}$, ha valutato l'effetto dell'auriculoterapia sul dolore, la funzionalità e la mobilità di individui con mal di schiena cronico. Diviso 31 partecipanti in tre gruppi: sperimentale con aghi di sangue inossidabili usa e getta di $1,5 \mathrm{~mm}$; sperimentale con semi di senape; controllo senza intervento. La scala analogica visiva è stata utilizzata per valutare il dolore; per il test di funzionalità, sono stati utilizzati il questionario sulla disabilità lombare del Quebec e il test di seduta in piedi; è stata eseguita la mobilità lombare.

Gli interventi sono stati eseguiti per 4 settimane, essendo una sessione settimanale, applicata nei punti: Shenmen, Rim, Sympathetic, Analgesia, Muscle Relaxation, 
Lumbar e Adrenal Spine (Figura 10). Dopo un intervallo di 1 settimana dopo l'ultima applicazione, gli individui sono stati rivalutati con lo stesso protocollo.

I risultati hanno mostrato un miglioramento nella condizione del mal di schiena, così come il miglioramento della capacità funzionale in entrambi i gruppi sperimentali, a differenza del controllo che non c'era alcuna differenza significativa in relazione al dolore iniziale. Non è stata riscontrata alcuna differenza tra le tecniche eseguite (ago e seme), indicando che entrambi sono benefici per il sollievo dal mal di schiena cronico e una maggiore capacità funzionale.

Figura 23: Punti dell'orecchio

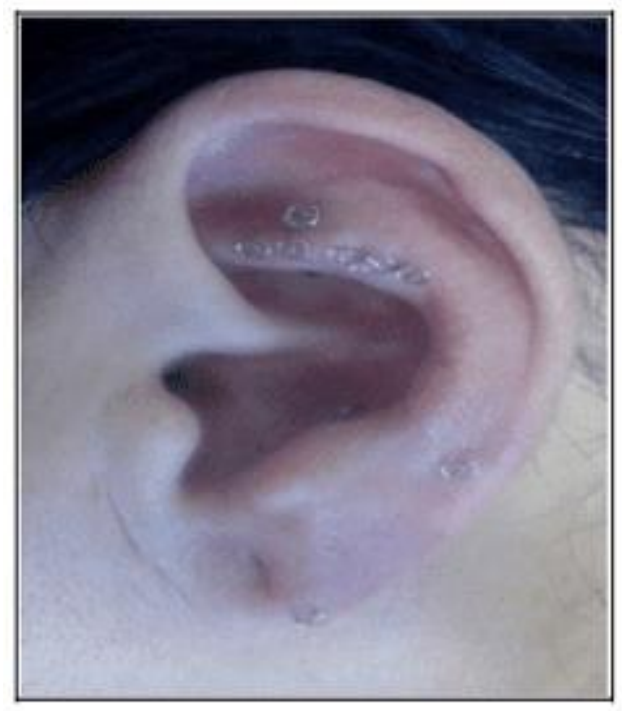

Fonte: Tolentino ${ }^{74}$

\section{CONSIDERAZIONI FINALI}

Sulla base della letteratura, analizzando tutti i risultati e le loro prove scientifiche, si ritiene che il trattamento dell'agopuntura sia efficace nel trattamento, nel miglioramento delle prestazioni e nella prevenzione delle lesioni negli atleti professionisti e dilettanti. Gli individui che ricevono l'intervento, anche se hanno un qualche tipo di complicanza ferita, il problema è meno grave e ripristina rapidamente la funzione organica, cioè 
l'atleta che subisce un certo tipo di infortunio e sta eseguendo l'agopuntura, questa avversità tenderà ad essere le attività minime e di ritorno anticipato.

Anche gli atleti che non usano l'agopuntura per prevenire la prevenzione e presentare la lesione, l'intervento di questo presenta prove cliniche, basate sulla neurofisiologia, il rilascio di oppioidi, con particolare attenzione al trattamento del dolore e al miglioramento del benessere del paziente .

L'intervento dell'agopuntura, anche se è una tecnica millenaria, è ancora in crescita in Occidente, ha ottenuto grandi risultati nella pratica clinica, nei temi della prevenzione delle lesioni muscolo-scheletriche, nonché nella prevenzione e nella cura delle malattie dolore psicosociale, acuto e cronico.

A causa dell'equilibrio del sistema energetico, l'agopuntura non tratta solo il benessere fisico, ma anche il benessere mentale, perché il fondamento teorico della tecnica non separa il corpo dalla mente beneficiando lo stress emotivo, riducendo l'ansia e bilanciando mente umana.

\section{RIFERIMENTI}

1. Ching N. O livro de ouro da medicina chinesa. 3. ed. Rio de Janeiro: Objetiva; 1991.

2. Malveira CL, Souza FCS, Rocha TBX. Biomecânica do movimento isométrico de pedala antes e após aplicação de acupuntura: Um estudo preliminar. Motric. 2012 Set;8(52):630-9.

3. Sousa NR. Relação entre a reatividade de pontos de acupuntura e atividade física com afecções ortopédicas determinadas por exames de imagens em equinos. Botucatu. Tese [Doutorado em Biotecnologia Animal] Universidade Estadual Paulista; 2015.

4. Silveira NC, Martins RCC. Eficácia da acupuntura para o tratamento da fibromialgia: Uma revisão sistemática. Psicol e Saúde em Debate. 2018 Fev; 4(1):85-105. 
5. Sant' Anna FM, Rangel VM, Mota DDS. Tratamento da Tenossinovite de De Quervain por Acupuntura - Relato de Caso. Vittalle. 2018 Mar; 30(1):130-6.

6. Tsuchiya M, Sato EF, Inoue M, Asada A. Acupuncture enhances generation of nitric oxide and increases local circulation. Anesth Analg. 2007 Feb; 104(2):3017.

7. Tavares MG, Machado AP, Motta BG, Borsatto MC, Rosa AL, Xavier SP. Electro-acupuncture efficacy on pain control after mandibular third molar sugery. Brz Dent J. 2007; 18(2):158-62

8. Hutchinson AJ, Ball S, Andrews JC, Jones GG. The effectiveness of acupuncture in theating chronic non-specific low back pain: a systematic review of the literature. J Otthop Surg Res. 2012 Oct 30;7:36.

9. Moniz PJL. Avaliação da acupuntura no desempenho do rugby. Coimbra. Dissertação [Mestrado Integrado em Medicina] - Faculdade de Medicina da Universidade de Coimbra; 2015.

10. Magalhães PC. Efeito da acupuntura na prevenção da hemorragia pulmonar induzida por exercício em cavalos puro sangue inglês de corrida. Botucatu. Dissertação [Mestrado em Biotecnologia Animal] - Universidade Estadual Paulista; 2016.

11. Matias B et al. Acupuntura de equilíbrio como coadjuvante no tratamento de disfunções temporomandibulares. Ação Odonto. 2018 Jun; 1(1):65-72.

12. Franco A. Acupuntura Ajuda Atletas nas Lesões e na Performance. Jornal da Madeira 2016 agosto 2;p.25.

13. Lopes SS, Mota MPG. Efeito da acupuntura na resistência física após exercícios repetitivos de tornozelo - Estudo experimental. R bras Ci e Mov. 2018 Jul;26(1):13-21.

14. Lopes SS, Mota MPG. Influência da acupuntura no limiar de percepção dolorosa de musculatura submetida a esforço repetitivo. Br J Pain. 2018 Jul; $1(3): 207-11$

15. Cohen M, Abdala RJ, Ejnisman B, Amaro JT. Lesões Ortopédicas no futebol, Rev. Bras. Ortop 1997 Dez;32(12):940-44.

16. Dias Junior JC, Marino DM. Acupuntura na prevenção de lesões musculares em atletas de futebol profissional. Rev Fisioter S Fun. Jan-Jul:6(1):36-42. 
17. Wen TS. Acupuntura Clássica Chinesa. 15. ed. São Paulo: Cultrix, 2006.

18. Maciocia G. Os fundamentos da medicina chinesa: um texto abrangente para acupunturistas e fitoterapeutas. São Paulo: Roca; 1996.

19. Ross J. Combinações dos Pontos de Acupuntura: A Chave para o Êxito Clínico. 1 ed. São Paulo: Roca, 2003.

20. Santos VC, Kawano MM, Banja RA. Acupuntura na melhora da performance em atletas juvenis de handebol, Rev Saúde e Pesq 2008 Set-Dez;1(3):331-35.

21. Rubio K, Godoy Moreira F. A dor em corredores com fascite plantar: o uso da acupuntura, Rev Dor 2008 Jul-Set;9(3):1290-1296.

22. Pires TF, Pellegrinotti IL. Acupuntura na Performance Atlética: Estudo Exploratório. [8 8 $^{\mathrm{a}}$ Mostra Acadêmica da UNIMEP; 2010 out; 1-4; Piracicaba, Brasil].

23. Yang HY, Liu TY, Gao M. Electrical acupoint stimulation increases athletes rapid strength, Zhongguo Zhen Jiu 2006 May;26(5): 313-315.

24. Luna MP, Fernandes Filho J. Efeitos da Acupuntura na performance de Atletas velocista de alto rendimento do Rio de Janeiro. Fit e Perform J 2005 Julh/Ago;4(4):199-214.

25. Ehrlich D, Haber P. Influence of acupuncture on pysical performance capacity and Haemodynamic Parameters. J. Sports Med 1992 May;13(6): 486-91.

26. França D, Fernandes-Senna V, Cortez CM. Acupuntura cinética como efeito potencializador dos elementos moduladores do movimento no tratamento de lesões desportivas. Fisioter Bras 2004 mar-abr; 5(2):111-8.

27. Wadsworth L T. Acupuncture in sports medicine. Curr Sports Med Rep 2006 Feb; 5(1):1-3.

28. Barela J A. Estratégias de Controle em Movimentos Complexos: Ciclo Percepção - Ação no Controle Postural. Rev Paul de Educ Fís 2000; 3: 79-88.

29. Papler PG et al. Reabilitação do joelho. In: Greve J.M. A. e Amattuzzi, M.M. Medicina de reabilitação aplicada à ortopedia e traumatologia. São Paulo: Roca, 1999.

30. Gemeo LH. Ignatti C. Acupuntura como Ferramenta Auxiliar do Aumento da Performance Desportiva. In: Anais do Simpósio Internacional de Ciências 
Integradas da UNAERP; 2004 ; Guarujá, Brasil. São Paulo: Universidade de Ribeirão Preto Campus Guarujá; 2004. p. 1-9.

31. Rossetto SC. Acupuntura nos Esportes. 1 ed. São Paulo: Phorte, 2009.

32. Pelham TW, Holt LE, Stalker R. Acupuncture in human Performance. J Strength Cond Res 2001 May;15(2):266-71.

33. Myamoto T. Acupuncture treatment for muscle injury. Japa Journ of Phys Fit and Spor Med 1997; 43(2):39-41.

34. Fry AC, Kraemer KJ. Resistance exercise overtraining and overreaching: neuroendocrine responses. Sports Med 1997 Feb;23(2): 106-29.

35. Frasca L. Desempenho na Ponta da Agulha. Rev Farmac 2011 out-nov:44-5.

36. Costa V. Acupuntura Previne Lesões e ajuda a melhorar o Desempenho nas Pistas [periódico na internet]. 2013 [acesso em 22 jan 2017] Disponível em: http://blogs.oglobo.globo.com/pulso/post/acupuntura-previne-lesoes-ajudamelhorar-desempenho-nas-pistas-500798.html.

37. Luna M. Os Benefícios da Acupuntura no Esporte. 2016 [acesso em 22 jan 2017] Disponível em: http://www.ibramrp.com.br/noticia/49/os-beneficios-daacupuntura-no-esporte.

38. Akimoto T, Nakahori C, Aizawa K, Kimura F, Fukubayashi T, Kono I. Acupuncture and responses of imunoligic and endocrine markers during competition, Med Sci in Sports Exerc 2003 Ago;35(8):1296-1302.

39. Bopp-Limoge C. L’acupuncture Permet-elle D`ameliorer les Performances Sportives Stude Personnelle a Propos de 35 Sportifs de haut niveau. These Medicine 1998; 117:215.

40. Lin ZP et al. Effects of acupuncture stimulation on recovery ability for male elite basketball athletes. Amer Jour of Chin Med 2009; 37(3):471-81.

41. Lin, ZP et al. Effect of auricular acupuncture on oxygen consumption of boxing athletes. Chin Med Jour 2009; 22(13):1587-90.

42. Dhillon $S$ et al. The acute effect of acupuncture on $20-\mathrm{km}$ cycling performance. Clin Jour of Spo Med 2008; 18(1):76-80. 
43. Hubscher $\mathrm{M}$ et al. Immediate effects of acupuncture on strength performance: a ramdomized, controlled crossover trial. Euro Jour of Appli Physi 2010; 110(2):353-58.

44. Geng $L \mathrm{~J}$ et al. Investigation on the effects of ear acupressure on exerciseinduced lactic acid levels and the implications for athletic training. Amer Jour of Acupu 1995; 23(4).

45. Fonseca LP, Lessa JFM. Efeito da Aplicação da Acupuntura na Resistência Muscular Localizada de Membros Superiores em Praticantes de Exercício Resistido. Brasília. Monografia [Graduação em Fisioterapia] - Centro Universitário de Brasília; 2011.

46. Belmiro H, Vicentini D, Camilotti CM. Efeitos da Acupuntura no Desempenho Motor de Atletas. Rev Fac Educ Fis 2013 jul-set; 11(3):176-91.

47. Angeli AL, Fernandes JGJ, Luna SPL. Acupuncture Applied Equine Sports Medicine, Ver Acad 2007 Jul-Set;5(3):325-33.

48. Staud R. Mechanisms of acupunture analgesia: effective therapy for musculoskeletal pain? Curr rheumatol Rep. 2007 Dec;9(6):473-81

49. Bucinskaite V, Lundeberg T, Stenfors C, Ekblom A, Dahlin L, Theodorsson E. Effects of electro-acupuncture and pysical exercice on regional concentrations of neuropeptides in rat brain, Brain research 1994 Dec;666(1): 128-32.

50. Shang C. Prospective tests on biological models of acupuncture. Evid Based Complement Alternat Med. 2009 Mar; 6(1):31-9.

51. Bowsher D. Mechanisms of acupuncture. In: Filshie J, White A. editors. Medial Acupuncture: a western scientific approach. London: Churchill Livingstone; 1988:69-82.

52. Ma SX. Neurobiology of acupuncture: Toward CAM. Evid Based Complement Alternat Med. 2004 Jun;1(1):41-7.

53. Li J, Wang Q, Liang H, Dong H, Li Y, Ng EH, et al. Biophysical characteristics of meridians and acupoints: a systematic review. Evid Based Complement Alternat Med. 2012;2012793841.

54. Cardeal EL, Oliveira KB, Aoki MN, Amarante MK, Oliveira CEC, Suzuki S, et al. Aspectos imunológicos da acupuntura. Biosaúde 2005 Dez:7(1/2):49-60. 
55. Szabó MVRS, Bechara GH. Acupuntura: Bases Científicas e Aplicações. Ciência rural 2001:31(6):1091-99

56. Saad M. A Medicina tradicional chinesa tem base científica?. Einstein: Educ Contin Saúde 2008: 6(3):122-6.

57. Alvarenga TF, Amaral CG, Steffen CP. Ação da acupuntura na neurofisiologia da dor: revisão bibliográfica. Rev Amazôn Scienc \& Helth 2014 Out/Dez:2(4):29-36.

58. Pereira FAO. Evidências científicas da ação da acupuntura. Perspectivas 2005 jan/jul:4(7):88-105.

59. Taffarel MO, Freitas PMC. Acupuntura e analgesia: aplicações clínicas e principais acupontos. Ciênc Rural 2009 Dez:39(9): 2665-72.

60. Lopes LF, Lopes MC, Fialho FAP, Gonçalves AL. Sistema de conhecimento para diagnóstico em acupuntura:uma modelagem usando o CommonKADS. Gest Prod 2010 Dez:x(x):1-15.

61.Zhu J, Arsovska B, Kozovska K. Acupuncture treatment for sports injury hamstring muscles group, Inter J Clin Exper Med Sci 2017 Nov;3(6):71-3.

62. Vickers AJ, Cronin AM, Maschino AC, Lewith G, MacPherson H, Foster NE, et al. Acupuncture for chronic pain individual patient data meta-analysis, Arch Intern Med 2012 Oct;172(19):1444-53.

63. Toda S. Effect of acupuncture on carnitine for skeletal muscle fatigue, Chin Med 2012 Jan;3:9-12.

64. Lopes SS, Mota MPG. Influência da acupuntura no limiar de percepção dolorosa de musculatura submetida a esforço repetitivo, $\mathrm{Br} \mathrm{J}$ Pain 2018 julset;1(3):207-11.

65. Lee, SH, Chung, SH, Lee, JS, Kim, SS, Shin, HD, Lim, BV, et al. Effects Acupunturaq on the 5-hydroxytryptamine synthesis and tryptophan hydroxylase expression in dorsal in the dorsal raphe of exercice rats. Neurocienses Letters 2002 Oct;332(1): 17-20.

66. Zyloney CE, Jensen K, Polich G, Loiotile RE, Cheetham A, LaViolette PS, et al. Imaging the functional connectivity of the Periaqueductal Gray during genuine and sham electroacupuncture treatment. Mol pain. 2010 Nov;6:80 
67. Minori AET, Mejia DPM. Atuação da Acupuntura para o Tratamento de LER/DORT no Ombro. Goiânia. Monografia [Pós Graduação em Acupuntura] Faculdade Ávila; 2007.

68. Brum KN, Alonso, AC, Brech GC. Tratamento de massagem e acupuntura em corredoresrecreacionais com síndrome do piriforme. Arq Cienc Sau 2009 abrjun; 16(2): 62-6.

69. Hongwen S. Clinical Observation on Acupuncture Treatment of Piriformis Syndrome. J Tradit Chin Med. 2003 mar; 23(1):38-9.

70. Rocha TBX, Vilela Junior GB, Martins GC, Manzatto L, Grande AJ. Análise Comparativa Eletromiográfica do Reto Femoral em Isometria na Posição Inferior do Agachamento Wall Slide, antes e após a Aplicação da Acupuntura no Ponto ST45. Rev Bras Cien e Mov 2012 jul; 20(4):92-8.

71. Pinheiro RG, Mejia DPM. Efeito da Acupuntura na Melhora do Paciente com Quadro Álgico de Lesão de Menisco Medial. Goiânia. Monografia [Pós Graduação em Acupuntura] - Faculdade Ávila; 2012.

72. Nunes EA, Mejia DPM. Tratamento de Acupuntura para Combater Dores nos Ombros. Goiânia. Monografia [Pós Graduação em Acupuntura] - Faculdade Ávila; 2012.

73. Parisotto D. Efeito Imediato da Aplicação da Acupuntura na Dor Muscular Tardia e na Capacidade de Contração Muscular. Curitiba. Dissertação [Mestrado em Fisiologia] - Universidade Federal do Paraná; 2014.

74. Tolentino F. Efeito de um Tratamento comAuriculoterapia na Dor, Funcionalidade e Mobilidade de Adultos com Dor Lombar Crônica. Rio Claro. Dissertação [Mestrado em Desenvolvimento Humano e Tecnologias] Universidade Estadual Paulista - UNESP; 2016.

Inviato: Aprile, 2019.

Approvato: ottobre 2019. 